\title{
A Wave Based Method for the efficient solution of the 2D poroelastic Biot equations
}

\author{
Elke Deckers ${ }^{\mathrm{a}, *}$, Nils-Erik Hörlin ${ }^{\mathrm{b}}$, Dirk Vandepitte ${ }^{\mathrm{a}}$, Wim Desmet $^{\mathrm{a}}$ \\ ${ }^{a}$ Department of Mechanical Engineering, Katholieke Universiteit Leuven, \\ 3001 Heverlee, Belgium \\ ${ }^{b}$ KTH Aeronautical and Vehicle Engineering, Marcus Wallenberg Laboratory of Sound and Vibration Research \\ 10044 Stockholm, Sweden
}

\begin{abstract}
The vibro-acoustic behaviour of poroelastic materials is often formulated as boundary value problems based on the continuum mechanics Biot's theory expressed as two coupled partial differential equations. This paper presents an extension of the Wave Based Method (WBM), a numerical technique to solve these vibro-acoustic boundary value problems in a computationally efficient manner. At present, the Finite Element Method (FEM) is the most commonly used prediction technique to deal with these Biot equations, but suffers from the disadvantage that the system matrices have to be recalculated for each frequency of interest due to the frequency-dependent equation parameters. This harms the inherent effectiveness of the FEM. Additionally, due to the discretisation into a large number of small finite elements and the high number of unknowns per node, the computational efforts involved practically restrict the use of FEM to low-frequency applications. The method discussed in this paper is based on an indirect Trefftz approach. Exact solutions of the three coupled waves, supported by Biot's equations, are used as basis functions in a solution expansion to approximate the field variables in a poroelastic boundary value problem. This approach leads to smaller systems of equations, enabling an efficient solution at higher frequencies.
\end{abstract}

Keywords: poroelastic materials, Biot theory, Wave Based Method, Trefftz

${ }^{*}$ Corresponding author, Tel.: +3216322594 ; Fax: +3216322987.
Email address: Elke.Deckers@mech.kuleuven.be (Elke Deckers) 


\section{Introduction}

Lightweight poroelastic materials are often used as efficient noise reduction measures in engineering applications such as automotive and aircraft interior noise [1, 2]. Considerable efforts have been put in the development of robust prediction techniques which are capable of accurately describing damping phenomena of these materials.

A poroelastic material consists of two phases, being the elastic solid phase and the fluid phase contained within the pores. Energy is dissipated in the material by structural, thermal and viscous means. The Biot theory [3], as adopted by Johnson [4], Champoux [5] and Allard [6] is most commonly used to model the fully coupled dynamic behaviour of such materials and leads to two coupled vectorial partial differential equations. This theory predicts the existence of three different types of propagating coupled waves, one shear wave and two longitudinal waves. Most of the material parameters, taking into account dissipation phenomena, are complex and frequency-dependent. The parameters in this model are defined on a macroscopic scale and cannot directly be related to microscopic parameters. Recently, Yamamoto et al. [7] derived macroscopic governing equations derived from microscopic structures using the homogenization method. Upscaling procedures using a statistical framework [8] may be used to replace highly heterogeneous porous media by an effective viscoelastic medium which behaves on the average as the original medium.

Finite Element formulations have been developed to account for absorbing media. Simplified approaches are often applied, such as impedance relations based on empirical laws, e.g. the work of Delany and Bazley [9], transfer matrix representations as described by Allard [10] and equivalent fluid formulations which only take into account one single propagating wave, see e.g. the work of Attenborough [11]. The Finite Element Method (FEM) has been used to model the fully coupled Biot equations based on different weak formulations, such as the $\left(\mathbf{u}^{\mathbf{s}}, \mathbf{u}^{\mathbf{f}}\right)$-formulation [12, 13] and the $\left(\mathbf{u}^{\mathbf{s}}, p\right)$-formulation $[14,15]$, with $\mathbf{u}^{\mathbf{s}}$ and $\mathbf{u}^{\mathbf{f}}$ the solid and fluid displacement vectors and $p$ the pore pressure in the fluid phase of the poroelastic material. A major advantage of the FE approach is that it poses almost no geometrical restrictions; complex geometries, heterogeneities, inclusions, etc. can all be tackled.

However, the use of the FEM often leads to time consuming calculations because of three reasons. Firstly, the FE matrices have to be recalculated for each frequency due to the frequencydependent equation parameters. Secondly, the number of unknowns per node is relatively high. Finally, since in many acoustic control applications the frame stiffness is low, the wavelengths 
are small. To resolve these, often extremely fine discretisations are needed. In addition, since the dimension of the computational domain is often large as compared to the wavelength, the numerical dispersion error, also known as pollution error, becomes significant, leading to even more restrictive requirements on the mesh discretisation [16]. As a consequence, in engineering applications, the FEM is often restricted to low-frequency calculations. Also, since the primary response variables are most commonly approximated using simple polynomial shape functions, the higher-order derived quantities are less accurately predicted than the primary ones. The standard FE formulations can be improved by using other formulations [17] and also through the use of hierarchical elements $[18,19]$. A generalised complex mode technique has been developed and applied for one [20] and three-dimensional cases [21]. It takes into account frequency non-linearities and leads to a reduction of the number of degrees of freedom, but with the current techniques the calculation of the modes is not a trivial task. Efficient sub-structuring based implementations apply the FEM to calculate the mechanical impedance of a trim-layer and combine this with the modal mechanical impedance of the basis plate. A combination with BEM models enables the prediction of the radiated power by the structure which can be used within large systems [6, 22].

Tanneau et al. [23] introduced the Boundary Element Method (BEM) for modelling porous media. In this case, only the boundary of the considered problem domain has to be discretised. Unlike FEM, BEM leads to a full system of matrix equations, obstructing the use of sparse solvers. Still, a large number of boundary elements is required due to the approximating nature of the applied shape functions, such that also the BEM is restricted to lower frequencies.

At higher frequencies, the Transfer Matrix Method (TMM) is widely used and validated to predict the transmission loss of multilayer structures [6]. Due to the infinite extent assumption, it is only valid at higher frequencies. Ghinet and Atalla [24] developed the Finite Transfer Matrix method (FTMM) to account for finite size effects occuring at lower frequencies. The FTMM has been applied to calculate transmission loss and absorption of flat finite multilayer structures [25]. Efficient FTMM formulations are given for acoustic as well as for structure-borne excitation and are compared to FEM-predictions, combined TMM-SEA-approaches and combined TMM-modal approaches in $[6,26]$. A drawback here is that all the layers have to be flat, such that the use of TM methods is restricted to flat geometries.

The Wave Based Method (WBM) [27] is an alternative deterministic method and is based on an indirect Trefftz approach $[28,29]$. In the WB modelling approach, the problem domain is 
partitioned into a small number of large convex subdomains. Convexity of the subdomains is a sufficient condition for the method to converge towards the exact solution of the problem [27]. In each subdomain, the dynamic response variables are approximated by a function series expansion of so-called wave functions, which are exact solutions of the governing differential equations. The degrees of freedom are the contribution factors of each wave function. Being exact solutions of the governing equations, the wave functions only violate the boundary and continuity conditions imposed on the boundaries of the subdomains. Enforcing the residuals on the boundaries and interfaces to be $L^{2}$-orthogonal to a finite set of test functions through a Galerkin weighted residual formulation yields a small matrix system of equations which can be solved for the contribution factor of each wave function. As compared to the FEM, the resulting numerical models are much smaller, with a faster convergence, enabling solutions for an extended frequency range. The derivatives of wave functions are wave functions with the same spatial resolution such that the formulation preserves a similar accuracy for both the primary and the secondary variables. WBM has been successfully applied for acoustic [30,31], equivalent fluid [32], uncoupled elastic [33, 34] and fully coupled vibro-acoustic problems $[35,36]$. The main drawback of the method is that the geometrical complexity of the problem domain has to be moderate to obtain efficient solutions, because otherwise a subdivision into a large number of convex subdomains is required or may even be impossible for some configurations, such as circular inclusions. Two recently developed approaches aim to relax on these limitations: a multi-level modelling framework allows the WBM to efficiently tackle configurations of multiple scatterers or inclusions $[37,38]$ and a hybrid FE-WBM formulation can be applied for complexly shaped domains [39].

This paper presents the extension of this modelling approach to solve the Biot equations more efficiently. The Biot equations can be decoupled into three Helmholtz equations in two different ways; one is in terms of longitudinal and rotational strains and the other one is based on scalar and vector potentials. The WB principle can then be applied to each of the three Helmholtz equations. In this way, the three aforementioned wave types existing in poroelastic materials are explicitly taken into account. Both formulations lead to the same expressions for the field variables, except for some scaling factors. The proposed approach leads to substantially smaller systems of equations as compared to FEM, allowing for more computationally efficient solutions of the Biot equations and can be applied in a broader frequency range. This paper is organised as follows. Section 2 shortly reviews the Biot equations with common boundary conditions and the material properties 
used throughout the paper. The WB approach to study the behaviour of two-dimensional isotropic poroelastic materials is explained in Section 3. Two different formulations are given, which are based on the strains and the potentials in poroelastic materials respectively. Also the strengths and weaknesses as compared to the FEM are discussed. Section 4 demonstrates the use of the WBM and its potential as compared to the FEM for geometrically simple numerical verification examples, considering different boundary conditions and different materials. The paper ends with some concluding remarks. Appendix A gathers and explains all symbols used within this paper.

\section{Problem definition}

The poroelastic materials considered in this paper are open cell materials, consisting of a solid phase $\left(\bullet^{s}\right)$, also known as the frame, and a fluid phase $\left(\bullet^{f}\right)$ filling the voids. The Biot theory [3] applies an equivalent solid and a compressible fluid continuum description on a macroscopic level. This is justified in the case that the characteristic dimensions of the material, e.g. the pore size, are small as compared to characteristic dimensions on the macroscopic level, typically the wavelengths of the different types of waves which propagate through the material [40,41]. The interaction between both phases is described using coupling parameters which are derived from measurable macroscopic properties. Fluid-structure interaction occurs throughout the whole material and the different waves can be strongly coupled.

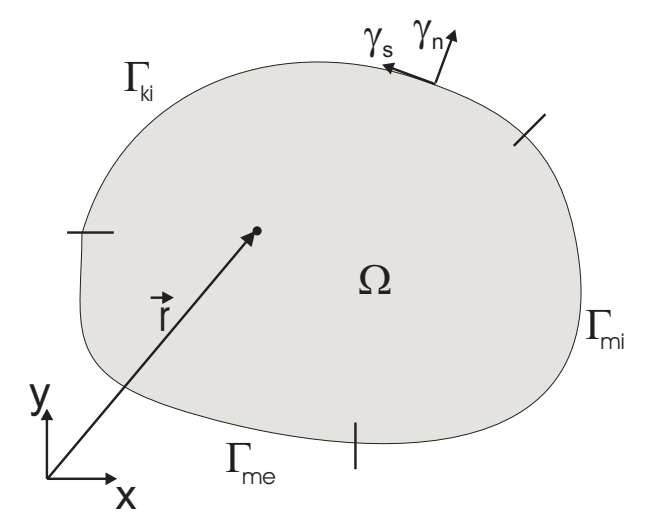

Figure 1: General description of a 2D poroelastic domain

Figure 1 shows a general 2D poroelastic domain $\Omega$, with various kinds of boundary conditions. A time-harmonic motion with $e^{\mathrm{i} \omega t}$-dependence is assumed, where $\mathrm{i}$ is the imaginary unit and $\omega=2 \pi f$ 
is the circular frequency and $t$ is the time. $\gamma_{n}$ and $\gamma_{s}$ are respectively the normal and the tangential vector to the boundary.

According to the Biot theory, the momentum equations can be written as:

$$
\begin{aligned}
& \nabla \cdot \boldsymbol{\sigma}^{\boldsymbol{s}}(\mathbf{r})=-\omega^{2} \rho_{1} \mathbf{u}^{\mathbf{s}}(\mathbf{r})-\left(\omega^{2} \rho_{a}-\mathrm{i} \omega b\right)\left(\mathbf{u}^{\mathbf{s}}(\mathbf{r})-\mathbf{u}^{\mathbf{f}}(\mathbf{r})\right), \\
& \nabla \cdot \boldsymbol{\sigma}^{\boldsymbol{f}}(\mathbf{r})=-\omega^{2} \rho_{2} \mathbf{u}^{\mathbf{f}}(\mathbf{r})-\left(\omega^{2} \rho_{a}-\mathrm{i} \omega b\right)\left(\mathbf{u}^{\mathbf{f}}(\mathbf{r})-\mathbf{u}^{\mathbf{s}}(\mathbf{r})\right),
\end{aligned}
$$

with $\boldsymbol{\sigma}^{\bullet}(\mathbf{r})$ the stress tensor and $\mathbf{u}^{\bullet}(\mathbf{r})$ is the displacement vector of phase $\bullet$. $\rho_{1}$ and $\rho_{2}=h \rho_{0}$ are respectively the bulk densities of the solid and the fluid phase and $\rho_{a}=h \rho_{0}\left(\alpha_{\infty}-1\right)$ is the inertial coupling term. $h$ is the porosity, $\rho_{0}$ is the density of the interstitial fluid and $\alpha_{\infty}$ is the tortuosity [4]. $b$ is a viscous coupling factor that can be related to the static macroscopic flow resistivity $\sigma$ of the porous material, $b=\sigma h^{2} G(\omega)$, taking into account the transition from microscale Stokes flow at very low frequencies to inviscid flow as high frequency asymptote. The constitutive relations are:

$$
\begin{aligned}
\boldsymbol{\sigma}^{s}(\mathbf{r}) & =\left[\lambda e^{s}(\mathbf{r})+Q e^{f}(\mathbf{r})\right] \mathbf{I}+2 N \mathbf{e}^{\mathbf{s}}(\mathbf{r}), \\
\boldsymbol{\sigma}^{f}(\mathbf{r}) & =\left[Q e^{s}(\mathbf{r})+R e^{f}(\mathbf{r})\right] \mathbf{I}
\end{aligned}
$$

where $e^{\bullet}(\mathbf{r})$ is the volumetric strain of phase $\bullet$, also known as dilatation and $\mathbf{e}^{\bullet}(\mathbf{r})$ indicates the strain tensor of phase $\bullet . \lambda=\frac{E \nu}{(1+\nu)(1-2 \nu)}$ is the first Lamé constant of the solid phase and $N$ is the second, the shear modulus of the solid phase. $E=E_{s}\left(1+\mathrm{i} \eta_{l}\right)$ is the in vacuo modulus of elasticity of the bulk solid phase and $\eta_{l}$ is the loss factor to take into account internal frictional losses. $Q$ is a dilatational coupling factor describing fluid stress dependence of frame dilatation and conversely frame stress dependence of fluid dilatation. The parameter $R$ can be interpreted as the fluid phase bulk stiffness at zero frame dilatation. Hörlin et al. [18] replace $\lambda$ in equation (3) by $\tilde{\lambda}+Q^{2} / R$. In this case $\tilde{\lambda}$ has to be interpreted as the Lamé parameter at zero acoustic pore pressure.

Expressions for the elastic constants can be found by using Biot's 'gedanken experiments' [42]. Appendix B gives an overview of the definitions of $b, G(\omega), Q$ and $R$ as they are used within the verification section.

By substituting the constitutive relations (3)-(4) into the momentum equations for the solid and the fluid phase (1)-(2), one obtains the well-known Biot equations, which are two coupled differential equations:

$$
\begin{aligned}
N \nabla^{2} \mathbf{u}^{\mathbf{s}}(\mathbf{r})+\nabla\left[(\lambda+N) e^{s}(\mathbf{r})+Q e^{f}(\mathbf{r})\right] & =-\omega^{2}\left(\rho_{11}^{*} \mathbf{u}^{\mathbf{s}}(\mathbf{r})+\rho_{12}^{*} \mathbf{u}^{\mathbf{f}}(\mathbf{r})\right), \\
\nabla\left[Q e^{s}(\mathbf{r})+R e^{f}(\mathbf{r})\right] & =-\omega^{2}\left(\rho_{12}^{*} \mathbf{u}^{\mathbf{s}}(\mathbf{r})+\rho_{22}^{*} \mathbf{u}^{\mathbf{f}}(\mathbf{r})\right),
\end{aligned}
$$


where $\rho_{11}^{*}=\rho_{11}+b / \mathrm{i} \omega, \rho_{11}=\rho_{1}+\rho_{a}, \rho_{12}^{*}=\rho_{12}-b / \mathrm{i} \omega, \rho_{12}=-\rho_{a}, \rho_{22}^{*}=\rho_{22}+b / \mathrm{i} \omega$ and $\rho_{22}=\rho_{2}+\rho_{a}$. The coefficients $\rho_{11}, \rho_{22}$ and $\rho_{12}$ are mass coefficients which take into account the inertial effects of the nonuniform fluid flow through the pores [3]. $\rho_{11}^{*}, \rho_{22}^{*}$ and $\rho_{12}^{*}$ take into account the total viscous and inertial energy dissipation caused by the relative motion between the solid and the fluid phase.

For a poroelastic material, three boundary conditions have to be specified at each point of the boundary in order to have a well-posed problem. As shown in Figure 1, different kinds of nonoverlapping boundaries $\Gamma_{k i}, \Gamma_{m e}$ and $\Gamma_{m i}$ can be considered along the surface $\Gamma\left(=\Gamma_{k i} \bigcup \Gamma_{m e} \bigcup \Gamma_{m i}\right)=$ $\delta \Omega$ of a poroelastic domain $\Omega$. For each kind of boundary, three residual error functions can be defined:

- kinematic boundary conditions along $\Gamma_{k i}$; the displacement components are prescribed:

$$
\mathbf{r} \in \Gamma_{k i}:\left\{\begin{array}{l}
R_{u_{n}^{s}}(\mathbf{r})=u_{n}^{s}(\mathbf{r})-\bar{u}_{n}^{s}(\mathbf{r})=0 \\
R_{u_{s}^{s}}(\mathbf{r})=u_{s}^{s}(\mathbf{r})-\bar{u}_{s}^{s}(\mathbf{r})=0 \\
R_{u_{n}^{f}}(\mathbf{r})=u_{n}^{f}(\mathbf{r})-\bar{u}_{n}^{f}(\mathbf{r})=0
\end{array}\right.
$$

with $\bar{u}_{n}^{s}(\mathbf{r}), \bar{u}_{s}^{s}(\mathbf{r})$ and $\bar{u}_{n}^{f}(\mathbf{r})$ respectively the prescribed values of the displacement components of the solid phase in the normal and tangential direction to the boundary and the prescribed value of the displacement of the fluid phase in the normal direction to the boundary.

- mechanical boundary conditions along $\Gamma_{m e}$; the stress resultants are prescribed:

$$
\mathbf{r} \in \Gamma_{m e}:\left\{\begin{array}{l}
R_{\sigma_{n}^{s}}(\mathbf{r})=\sigma_{n}^{s}(\mathbf{r})-\bar{\sigma}_{n}^{s}(\mathbf{r})=0 \\
R_{\sigma_{s}^{s}}(\mathbf{r})=\sigma_{s}^{s}(\mathbf{r})-\bar{\sigma}_{s}^{s}(\mathbf{r})=0 \\
R_{\sigma^{f}}(\mathbf{r})=\sigma^{f}(\mathbf{r})-\bar{\sigma}^{f}(\mathbf{r})=0
\end{array}\right.
$$

with $\bar{\sigma}_{n}^{s}(\mathbf{r}), \bar{\sigma}_{s}^{s}(\mathbf{r})$ and $\bar{\sigma}^{f}(\mathbf{r})$ respectively the prescribed values of the normal and tangential stress resultant components of the solid phase in the normal and tangential direction to the boundary and the prescribed hydrodynamic stress of the fluid phase.

- mixed boundary conditions along $\Gamma_{m i}$ :

$$
\mathbf{r} \in \Gamma_{m i}:\left\{\begin{array}{l}
R_{u_{n}^{s}}(\mathbf{r})=u_{n}^{s}(\mathbf{r})-\bar{u}_{n}^{s}(\mathbf{r})=0 \\
R_{u_{n}^{f}}(\mathbf{r})=u_{n}^{f}(\mathbf{r})-\bar{u}_{n}^{f}(\mathbf{r})=0 \\
R_{\sigma_{s}^{s}}(\mathbf{r})=\sigma_{s}^{s}(\mathbf{r})-\bar{\sigma}_{s}^{s}(\mathbf{r})=0
\end{array}\right.
$$


For a sliding edge boundary condition [15], the normal displacements are constrained and the tangential displacements of the solid phase are free. The prescribed values of $\bar{u}_{n}^{s}(\mathbf{r}), \bar{u}_{n}^{f}(\mathbf{r})$ and $\bar{\sigma}_{s}^{s}(\mathbf{r})$ are zero.

The Biot equations (5)-(6) together with the associated boundary conditions (7), (8) and (9) define unique fields in the solid and the fluid phase.

\section{The Wave Based Method for poroelastic materials}

\subsection{Basic concept}

The WBM [27] is a deterministic numerical method for solving boundary value problems in e.g. vibro-acoustics, aiming at relaxing the frequency limitations imposed by the element-based approaches, such as FEM and BEM. The WBM is based on an indirect Trefftz approach [28] in that it applies exact solutions of the governing differential equations. WBM renders solutions of the boundary value problem which exactly satisfy the differential equations, but satisfy the boundary conditions only in an approximate way. As a result, the use of very fine domain discretisations as for the FEM is not longer required, leading to smaller model sizes and associated computational cost. The WB modelling approach generally consists of four steps, which will be applied for the Biot equations:

1. Partitioning of the considered problem domain into convex subdomains

2. Selection of a suitable set of wave functions for each subdomain

3. Construction of the WB system matrices using a weighted residual formulation of the boundary and interface conditions

4. Solution of the system of equations, yielding the wave function contribution factors and postprocessing of the dynamic variables

\subsection{Partitioning into convex subdomains}

A sufficient condition for the WBM to converge towards the exact solution of the problem is the convexity of the considered problem domain [27]. In a general poroelastic problem, the domain $\Omega$ may be non convex. A non-convex domain $\Omega$ has to be divided into $N_{\Omega}$ non-overlapping

convex subdomains $\Omega^{(\alpha)}, \alpha=1, \ldots, N_{\Omega}$. Also when different poroelastic materials are present, a corresponding division into subdomains is required. To ensure continuity along the poroelastic 
interfaces $\Gamma_{I}^{(\alpha, \beta)}$ between two subdomains $\Omega^{(\alpha)}$ and $\Omega^{(\beta)}$, continuity conditions between the approximations in each of the subdomains need to be explicitly imposed. Since in both subdomains, three decoupled Helmholtz equations are considered, six continuity conditions have to be imposed. This leads to the following six residuals [6]:

$$
\mathbf{r} \in \Gamma_{I}^{(\alpha, \beta)}:\left\{\begin{array}{l}
R_{u_{n}^{s}}^{(\alpha, \beta)}(\mathbf{r})=u_{n}^{s(\alpha)}(\mathbf{r})+u_{n}^{s(\beta)}(\mathbf{r})=0, \\
R_{u_{s}^{s}}^{(\alpha, \beta)}(\mathbf{r})=u_{s}^{s(\alpha)}(\mathbf{r})+u_{s}^{s(\beta)}(\mathbf{r})=0, \\
R_{u_{n}^{f}}^{(\alpha, \beta)}(\mathbf{r})=h^{(\alpha)}\left(u_{n}^{f(\alpha)}(\mathbf{r})-u_{n}^{s(\alpha)}(\mathbf{r})\right)+h^{(\beta)}\left(u_{n}^{f(\beta)}(\mathbf{r})-u_{n}^{s(\beta)}(\mathbf{r})\right)=0, \\
R_{\sigma_{n}^{s}}^{(\alpha, \beta)}(\mathbf{r})=\left(\sigma_{n}^{s(\alpha)}(\mathbf{r})+\sigma^{f(\alpha)}(\mathbf{r})\right)-\left(\sigma_{n}^{s(\beta)}(\mathbf{r})+\sigma^{f(\beta)}(\mathbf{r})\right)=0, \\
R_{\sigma_{s}^{s}}^{(\alpha, \beta)}(\mathbf{r})=\sigma_{s}^{s(\alpha)}(\mathbf{r})-\sigma_{s}^{s(\beta)}(\mathbf{r})=0, \\
R_{\sigma^{f}}^{(\alpha, \beta)}(\mathbf{r})=\frac{\sigma^{f(\alpha)}(\mathbf{r})}{h^{(\alpha)}}-\frac{\sigma^{f(\beta)}(\mathbf{r})}{h^{(\beta)}}=0 .
\end{array}\right.
$$

The first three conditions are imposed on subdomain $\Omega^{(\alpha)}$, the last three on subdomain $\Omega^{(\beta)}$. Together with the boundary conditions (7)-(9), three boundary conditions are defined on each point of the boundary of each subdomain $\Omega^{(\alpha)}$ leading to a well-posed problem description.

\subsection{Wave function selection}

Contrary to the FEM, which commonly uses polynomials with local support over an element, the WBM describes the field variables as an expansion of wave functions which are exact solutions of the governing differential equations. As noted by Biot [3], porous materials can sustain three wave types simultaneously, two longitudinal waves and one shear wave. Within the WB approach these three wave types are explicitly taken into account. If the material is isotropic, two possible decompositions are considered for the solid displacements:

1. By applying the divergence operation and the curl operation to the Biot equations [43], the displacement field can be decomposed into two dilatational strains, $e_{1}^{s}(\mathbf{r})$ and $e_{2}^{s}(\mathbf{r})$, and a rotational strain, $\boldsymbol{\Omega}^{\mathbf{s}}(\mathbf{r})=\Omega^{s}(\mathbf{r}) \cdot \mathbf{e}_{\mathbf{z}}$, with $\mathbf{e}_{\mathbf{z}}$ the unit vector normal to the considered $\mathrm{xy}$ plane:

$$
\left\{\begin{array}{l}
u_{x}^{s}(\mathbf{r}) \\
u_{y}^{s}(\mathbf{r})
\end{array}\right\}=\nabla\left(-\frac{1}{k_{l_{1}}^{2}} e_{1}^{s}(\mathbf{r})-\frac{1}{k_{l_{2}}^{2}} e_{2}^{s}(\mathbf{r})\right)+\nabla \times \frac{1}{k_{t}^{2}} \mathbf{\Omega}^{\mathbf{s}}(\mathbf{r})
$$

2. The second transformation is based on the Helmholtz decomposition of a vector field [44] which states that any vector field can be decomposed into an irrotational and a solenoidal 
part, provided that this vector field is piecewise differentiable. The derivation is given in [6] and leads to a decomposition into two scalar potentials, $\varphi_{1}^{s}(\mathbf{r})$ and $\varphi_{2}^{s}(\mathbf{r})$, and a vector potential, $\psi^{s}(\mathbf{r})=\psi^{s}(\mathbf{r}) \cdot \mathbf{e}_{\mathbf{z}}$ :

$$
\left\{\begin{array}{l}
u_{x}^{s}(\mathbf{r}) \\
u_{y}^{s}(\mathbf{r})
\end{array}\right\}=\nabla\left(\varphi_{1}^{s}(\mathbf{r})+\varphi_{2}^{s}(\mathbf{r})\right)+\nabla \times \boldsymbol{\psi}^{s}(\mathbf{r}) .
$$

By substituting either (11) or (12) in the Biot equations (5)-(6), one obtains the following set of decoupled Helmholtz equations:

$$
\begin{array}{r}
\left(\nabla^{2} \varsigma_{1}^{s}(\mathbf{r})+k_{l_{1}}^{2} \varsigma_{1}^{s}(\mathbf{r})\right)\left(\nabla^{2} \varsigma_{2}^{s}(\mathbf{r})+k_{l_{2}}^{2} \varsigma_{2}^{s}(\mathbf{r})\right)=0 \\
\nabla^{2} \chi^{s}(\mathbf{r})+k_{t}^{2} \chi^{s}(\mathbf{r})=0
\end{array}
$$

in which $\varsigma^{s}(\mathbf{r})=\varsigma_{1}^{S}(\mathbf{r})+\varsigma_{2}^{S}(\mathbf{r})$ is either the steady-state volumetric strain $e^{s}(\mathbf{r})$ or the scalar potential $\varphi^{s}(\mathbf{r})$ and $\chi^{s}(\mathbf{r})$ is either the steady-state rotational strain $\Omega^{s}(\mathbf{r})$ or the vector potential $\psi^{s}(\mathbf{r})$. Equation (13) indicates the existence of two dilatational wave types with distinct wave numbers $k_{l_{1}}$ and $k_{l_{2}}$ :

$$
\left\{\begin{array} { l } 
{ k _ { l _ { 1 } } = \sqrt { \frac { A _ { 1 } } { 2 } + \sqrt { \frac { A _ { 1 } ^ { 2 } } { 4 } - A _ { 2 } } } , } \\
{ k _ { l _ { 2 } } = \sqrt { \frac { A _ { 1 } } { 2 } - \sqrt { \frac { A _ { 1 } ^ { 2 } } { 4 } - A _ { 2 } } } , }
\end{array} \quad \text { with } \left\{\begin{array}{l}
A_{1}=\omega^{2} \frac{\rho_{11}^{*} R-2 \rho_{12}^{*} Q+\rho_{22}^{*}(\lambda+2 N)}{R(\lambda+2 N)-Q^{2}}, \\
A_{2}=\omega^{4} \frac{\rho_{11}^{*} \rho_{22}^{*}\left(\rho_{12}^{*}\right)^{2}}{R(\lambda+2 N)-Q^{2}}
\end{array}\right.\right.
$$

Equation (14) indicates the existence of one rotational wave type with wave number $k_{t}$ :

$$
k_{t}=\omega \sqrt{\frac{\rho_{11}^{*} \rho_{22}^{*}-\left(\rho_{12}^{*}\right)^{2}}{N \rho_{22}^{*}}} .
$$

The three fields $\varsigma_{1}^{s}(\mathbf{r}), \varsigma_{2}^{s}(\mathbf{r})$ and $\chi^{s}(\mathbf{r})$ can now be approximated in each subdomain $\Omega^{(\alpha)}$ according to the WB principle. Each of these three variables is approximated by a solution expansion in terms of $n_{\bullet}^{(\alpha)}$ wave functions $\Phi_{w_{\bullet}}^{(\alpha)}\left(w_{\bullet}=1, \ldots, n_{\bullet}^{(\alpha)}\right)$ :

$$
\left\{\begin{array}{c}
\varsigma_{1}^{s^{(\alpha)}}(\mathbf{r}) \simeq \hat{\varsigma}_{1}^{s^{(\alpha)}}(\mathbf{r})=\sum_{w_{\varsigma_{1}}=1}^{n_{\varsigma_{1}}^{(\alpha)}} u_{w_{\varsigma_{1}}}^{(\alpha)} \Phi_{w_{\varsigma_{1}}}^{(\alpha)}(\mathbf{r})=\Phi_{\mathbf{w}_{\varsigma_{1}}}^{(\alpha)}(\mathbf{r}) \mathbf{u}_{\mathbf{w}_{\varsigma_{1}}}^{(\alpha)}, \\
\varsigma_{2}^{s^{(\alpha)}}(\mathbf{r}) \simeq \hat{\varsigma}_{2}^{s^{(\alpha)}}(\mathbf{r})=\sum_{w_{\varsigma_{2}}=1}^{n_{\varsigma_{2}}^{(\alpha)}} u_{w_{\varsigma_{2}}}^{(\alpha)} \Phi_{w_{\varsigma_{2}}}^{(\alpha)}(\mathbf{r})=\Phi_{\mathbf{w}_{\varsigma_{2}}}^{(\alpha)}(\mathbf{r}) \mathbf{u}_{\mathbf{w}_{\varsigma_{2}}}^{(\alpha)} \\
\chi^{s^{(\alpha)}}(\mathbf{r}) \simeq \hat{\chi}^{s^{(\alpha)}}(\mathbf{r})=\sum_{w_{\chi}=1}^{n_{\chi}^{(\alpha)}} u_{w_{\chi}}^{(\alpha)} \Phi_{w_{\chi}}^{(\alpha)}(\mathbf{r})=\Phi_{\mathbf{w}_{\chi}}^{(\alpha)}(\mathbf{r}) \mathbf{u}_{\mathbf{w}_{\chi}}^{(\alpha)}
\end{array}\right.
$$

The wave function contribution factors $u_{w_{\bullet}}^{(\alpha)}$ belonging to each of the wave functions are gathered in the vectors of degrees of freedom $\mathbf{u}_{\mathbf{w}_{\bullet}}^{(\alpha)}$. The row vectors $\boldsymbol{\Phi}_{\mathbf{w}_{\bullet}}^{(\alpha)}(\mathbf{r})$ collect the $n_{\bullet}^{(\alpha)}$ wave functions 
$\Phi_{w_{\bullet}}^{(\alpha)}(\mathbf{r})$. Each wave function $\Phi_{w_{\bullet}}^{(\alpha)}(\mathbf{r})$ exactly satisfies the corresponding homogeneous Helmholtz equation in (13)-(14). For two-dimensional bounded domains, four sets of wave functions are distinguished, indicated by superscripts $a, b, c$ and $d$.

$$
\sum_{w_{\bullet}=1}^{n_{\bullet}^{(\alpha)}} u_{w_{\bullet}}^{(\alpha)} \Phi_{w_{\bullet}}^{(\alpha)}(\mathbf{r})=\sum_{w_{\bullet}=1}^{n_{\bullet}^{a(\alpha)}} u_{w_{\bullet}}^{a(\alpha)} \Phi_{w_{\bullet}}^{a(\alpha)}(\mathbf{r})+\sum_{w_{\bullet}=1}^{n_{\bullet}^{b(\alpha)}} u_{w_{\bullet}}^{b(\alpha)} \Phi_{w_{\bullet}}^{b(\alpha)}(\mathbf{r})+\sum_{w_{\bullet}=1}^{n_{\bullet}^{c(\alpha)}} u_{w_{\bullet}}^{c(\alpha)} \Phi_{w_{\bullet}}^{c(\alpha)}(\mathbf{r})+\sum_{w_{\bullet}=1}^{n_{\bullet}^{d(\alpha)}} u_{w_{\bullet}}^{d(\alpha)} \Phi_{w_{\bullet}}^{d(\alpha)}(\mathbf{r})
$$

with $n_{\bullet}^{(\alpha)}=n_{\bullet}^{a(\alpha)}+n_{\bullet}^{b(\alpha)}+n_{\bullet}^{c(\alpha)}+n_{\bullet}^{d(\alpha)}$. can be $\varsigma_{1}, \varsigma_{2}$ or $\chi$. The wave functions are defined as:

$$
\left\{\begin{array}{c}
\Phi_{w_{\bullet}}^{a(\alpha)}(x, y)=\sin \left(k_{x w_{\bullet}}^{a(\alpha)} x\right) e^{-\mathrm{i} k_{y w_{\bullet}}^{a(\alpha)} y} \\
\Phi_{w_{\bullet}}^{b(\alpha)}(x, y)=\cos \left(k_{x w_{\bullet}}^{b(\alpha)} x\right) e^{-\mathrm{i} k_{y w_{\bullet}}^{b(\alpha)} y} \\
\Phi_{w_{\bullet}}^{c(\alpha)}(x, y)=e^{-\mathrm{i} k_{x w_{\bullet}}^{c(\alpha)} x} \sin \left(k_{y w_{\bullet}}^{c(\alpha)} y\right) \\
\Phi_{w_{\bullet}}^{d(\alpha)}(x, y)=e^{-\mathrm{i} k_{x w_{\bullet}}^{d(\alpha)} x} \cos \left(k_{y w_{\bullet}}^{d(\alpha)} y\right)
\end{array}\right.
$$

The only requirement for these wave functions to be exact solutions of one of the three Helmholtz equations in (13)-(14) is that the wave number components in (19) have to satisfy the associated dispersion relation:

$$
\begin{aligned}
& \left(k_{x w_{\bullet}}^{a(\alpha)}\right)^{2}+\left(k_{y w_{\bullet}}^{a(\alpha)}\right)^{2}=\left(k_{x w_{\bullet}}^{b(\alpha)}\right)^{2}+\left(k_{y w_{\bullet}}^{b(\alpha)}\right)^{2}= \\
& \left(k_{x w_{\bullet}}^{c(\alpha)}\right)^{2}+\left(k_{y w_{\bullet}}^{c(\alpha)}\right)^{2}=\left(k_{x w_{\bullet}}^{d(\alpha)}\right)^{2}+\left(k_{y w_{\bullet}}^{d(\alpha)}\right)^{2}=k_{j}^{2},
\end{aligned}
$$

with $k_{j}$ one of the three wave numbers, $k_{l_{1}}, k_{l_{2}}$ or $k_{t}$ as defined by equations (15)-(16). In a similar manner as is done for acoustic and vibro-acoustic problems [27], it is proposed here to select the following wave number components:

$$
\begin{aligned}
& \left(k_{x w_{\bullet}}^{a(\alpha)}, k_{y w_{\bullet}}^{a(\alpha)}\right)=\left(k_{x w_{\bullet}}^{b(\alpha)}, k_{y w_{\bullet}}^{b(\alpha)}\right)=\left(\frac{w_{\bullet, 1}^{(\alpha)} \pi}{L_{x}^{(\alpha)}}, \pm \sqrt{k_{j}^{2}-\left(k_{x w_{\bullet}}^{a(\alpha)}\right)^{2}}\right), \quad w_{\bullet, 1}^{(\alpha)}=0,1,2, \ldots \\
& \left(k_{x w_{\bullet}}^{c(\alpha)}, k_{y w_{\bullet}}^{c(\alpha)}\right)=\left(k_{x w_{\bullet}}^{d(\alpha)}, k_{y w_{\bullet}}^{d(\alpha)}\right)=\left( \pm \sqrt{k_{j}^{2}-\left(k_{y w_{\bullet}}^{c(\alpha)}\right)^{2}}, \frac{w_{\bullet, 2}^{(\alpha)} \pi}{L_{y}^{(\alpha)}}\right), \quad w_{\bullet, 2}^{(\alpha)}=0,1,2, \ldots
\end{aligned}
$$

The constants $L_{x}^{(\alpha)}$ and $L_{y}^{(\alpha)}$ are the dimensions of the smallest bounding rectangle circumscribing the considered subdomain $\Omega^{(\alpha)}$ as shown by Figure 2. Desmet [27] has shown that this set of wave functions forms a complete set and that the WBM will converge towards the exact solution of the problem given that the subdomains are convex.

This infinite set of wave functions has to be truncated into a finite set in order to apply the WBM 


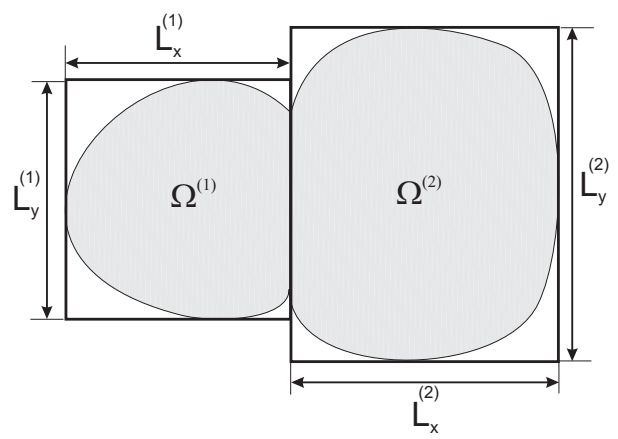

Figure 2: Illustration of the enclosing bounding box dimensions

in a numerical scheme. The upper limit $w_{\bullet, \star}^{(\alpha)}$ in the wave number selection (21)-(22) is chosen accordingly to the following truncation rule:

$$
\frac{w_{\bullet, \star}^{(\alpha)} \pi}{L_{\star}^{(\alpha)}} \geqslant T \max _{j}\left(k_{j}\right) .
$$

The physical wave number $k_{j}$ corresponds to the largest physical wave number in the problem domain. By using this truncation rule, the approximation fields in adjacent subdomains exhibit a similar spatial resolution along the interfaces. $T$ is a user defined truncation factor. Typically, $1 \leq T \leq 6$.

\subsection{Construction of the WB system matrices}

With the use of the proposed solution expansions (18), the three Helmholtz equations are always exactly satisfied, irrespective of the values of the unknown wave function contribution factors $u_{w_{\bullet}}$. These functions may however violate the imposed boundary and interface conditions. The residuals on the boundaries and interfaces of the subdomains, given by equations (7)-(9) and (10), are minimized in an integral sense using a Galerkin weighted residual formulation, expanding the weighting functions in a similar fashion as the field variables. This yields an algebraic equation for each subdomain, linking together the unknown contribution factors for each subdomain to those of the adjacent subdomains. Enforcing that these equations should hold for any combination of weighting functions results in a matrix system of equations which can be solved for the unknown contribution factors of each wave function in each domain $\Omega^{(\alpha)}$. More detail concerning the field variable expansions and the weighted residual formulation to obtain the system matrix is given in Appendix C. The calculation of the matrix coefficients involves integrations of highly oscillatory 
wave functions and is computationally more demanding than the construction of FE matrices. Like any Trefftz-method, the WBM suffers from a poor numerical condition [45, 46]. Consequently, a sufficiently high accuracy is required in the construction of the matrices. The application of the classical Gauss-Legendre integration rule, with a constant number of integration points per oscillatory wavelength of the integrand, was shown to be the most efficient approximation for evaluating the WB system matrices $[47,48]$ and is also applied here.

\subsection{Solution of the system of equations and postprocessing}

The fourth step in the WB modelling process is the solution of the WB system matrices (C.18)

for the unknown contribution factors of the wave functions in each subdoman $\Omega^{(\alpha)}$. The backsubstitution of the resulting wave function contribution factors into the field variable expansions (17), yields an approximation of the dynamic field variables. Derived variables can be computed by applying the corresponding differential operators (C.4) to the wave function sets combined with the calculated contribution factors.

\subsection{WBM Model properties and comparison with FEM}

Both the FEM and the WBM belong to the family of deterministic approaches, but differ in the choice of approximation functions and domain partitioning strategies. This results in different specific properties, advantages and disadvantages which are listed below:

Approximation functions and derived variables: In a FE model the degrees of freedom (dofs) are the nodal values whereas in a WB model the unknown wave function contribution factors form the dofs. The FEM generally applies approximating (mostly simple polynomial) shape functions to describe the field variables. Within the WBM exact solutions of the governing equations are used, greatly reducing the effects of numerical pollution. Due to the oscillatory nature of the wave functions, derivatives preserve the oscillatory nature exhibiting the same wavelength, allowing derived quantities to be determined without an additional loss in prediction accuracy.

Problem discretisation: Due to the simple approximation functions used in FEM, the domain needs to be discretised into a large number of small elements to capture the dynamics of the field variables. When frequency increases, the mesh needs to be refined to retain the same accuracy. As for the WBM, a sufficient condition for the convergence of the method is the use of convex subdomains [27]. In order to optimise the computational efficiency of the 
method, the number of subdomains should be as small as possible. The size and the shape of the subdomains is frequency-independent. In order to obtain a similar accuracy at higher frequencies, the number of wave functions in each subdomain is increased.

Problem geometric complexity: Because of the fine discretisation of the FEM, it has almost no restrictions regarding geometric complexity. Complex shapes can easily be modelled. Contrarily, problems with a complex geometry are hard to model with the WBM. When the geometry of the problem domain becomes more complex, a larger number of small subdomains is required to respect the convexity requirement. In order to couple all subdomains together, a large number of interface conditions has to be imposed leading to an increased cost for the evaluation of the boundary integrals in the construction of the coupling matrices. This imposes limitations on the geometrical complexity for which the WBM can be applied. Two recent developments partially relax those constraints and increase the application range of the method. Some geometries, like a cavity containing an inclusion inherently lead to a partitioning into a large number of subdomains and the calculations can be infeasible. These limitations are alleviated by a so-called multi-level modelling framework [37, 38]. Another approach towards relaxing the geometrical constraints is the hybrid FE-WBM approach [39]. It combines the ability of the FEM to handle the geometrically complex parts of the problem domain and the WBM to deal in a more efficient way with the large, convex parts of the problem domains. This has been applied for purely acoustic and vibro-acoustic problems. With these recent enhancements, the WBM is capable to deal with a wide range of dynamic problems and it is expected that these developments will soon be extended for poroelastic problems.

Heterogeneities, inclusions and anisotropy: Within the FEM, these types of problems can be tackled since different material properties can be attributed to each element. Fine meshes are required near heterogeneities and inclusions to accurately couple the behaviour of different media which can be costly to model. The two decompositions of the Biot equations (11)-(12) to obtain the three wave types used within the WBM demand the material to be isotropic. Similar decompositions are not possible for orthotropic or anisotropic materials and can not be dealt with, contrary to the FEM. Heterogeneities of complex shapes require a subdivision into a large number of subdomains which will hamper the efficient WB solution. Heterogeneities 
with simple shapes and voids can possibly be tackled applying the multi-level concept.

System matrix properties: Concerning the FEM, the system matrices are large and sparsely populated with a banded structure, allowing for efficient solution. Nevertheless, for poroelastic materials, the material properties are complex and frequency dependent. In this case, the FE matrices have to be recalculated for each frequency and are complex, hampering the efficient solution and limiting the practical frequency range. In contrast, the WB matrices are always complex, frequency dependent and fully populated. Hence, complex and frequency dependent material properties do not adversely affect the properties of the system matrices, which may be an advantage when coupling poroelastic domains to acoustic or elastic domains. As compared to the FEM, the WB matrices are very small, but like any Trefftz method also the WBM yields ill-conditioned system matrices $[45,46]$. Consequently, care has to be taken in the WB model construction. The numerical integrations to build the matrices must be performed carefully, ensuring a sufficiently high accuracy in determining the matrix coefficients. The largest computational effort of the WBM is related to the construction of the system matrices $[49,50]$. In the past, several analytical and numerical integration techniques have been studied [48]. Numerical integration, applying the Gauss-Legendre integration rule was shown to be the most efficient for the kind of integrals to be solved for a WB scheme, since an efficient matrix multiplication [47] can be applied. This numerical integration technique is applied with a frequency-dependent number of quadrature points per oscillatory wavelength of the integrand.

Computational performance: Because of the moderate WB model sizes, the solution of the system equations can be performed efficiently. The solution of the FE models takes much more time than the solution of the WB matrices, due to the large number of $\mathrm{FE}$ degrees of freedom. Moreover, the WBM exhibits a high convergence rate and as a result may tackle problems for an increased frequency range.

\section{Verification examples}

This section shows the potential of the newly developed WBM for three geometrically simple verification cases. The problem settings include different geometries, materials and boundary 


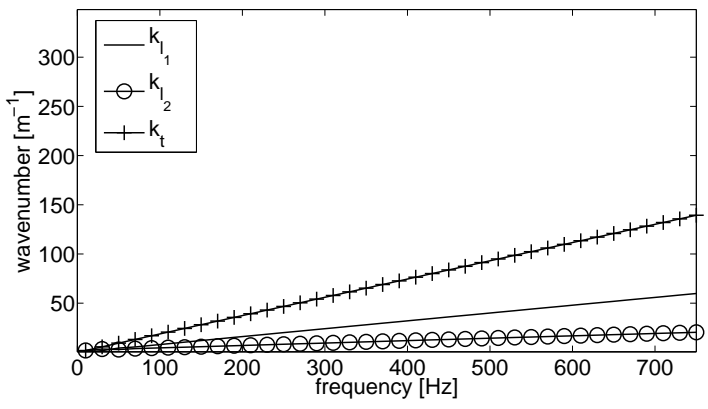

(a) Material 1

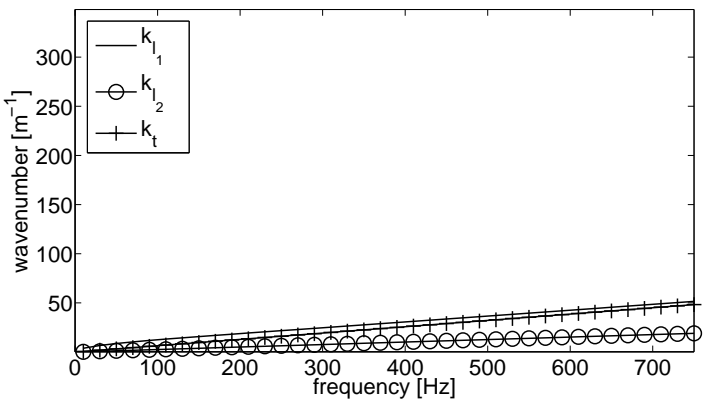

(b) Material 2

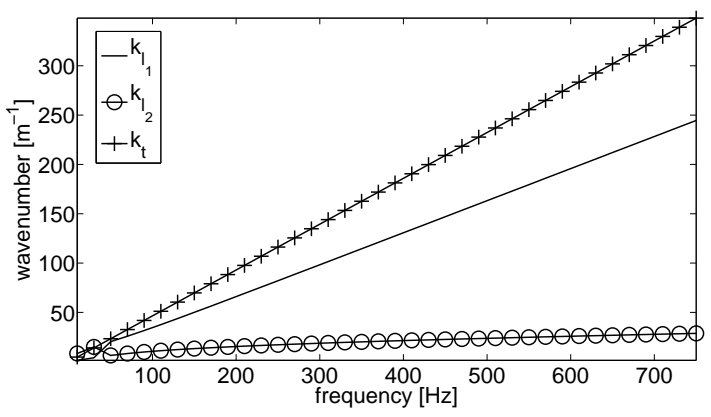

(c) Material 3

Figure 3: Absolute value of the wave numbers occuring in the three poroelastic materials as function of frequency

conditions. Table 1 contains the material data of the three considered materials. Material 1 is a polyurethane foam with an open cell structure and the first formulation of Appendix B is applied. According to the paper of Hörlin et al. [18] $\lambda$ in equation (3) is replaced by $\tilde{\lambda}+Q^{2} / R$. Material 2 is a partially reticulated polyurethane foam [43] and the second formulation of Appendix B is used. Material 3 is a part of a carpet and was applied in validation B of [15]. Figures 3(a) to 3(c) show the absolute value of the wave numbers of the three wave types, equations (15)-(16), for the three materials in function of frequency which gives an indication of the spectral content of each wave type.

This section evaluates both the strain and the potential formulation of the WBM and compares the predictions to results obtained with the FEM. This comparison is made in terms of calculation time as well as accuracy. All calculations are performed on a Linux-based 2.66 GHz Intel Xeon system. The WB routines are implemented in Matlab R2010a. The FE predictions are obtained using COMSOL3.5a, a commercially available software package, capable of dealing with weak integral 


\begin{tabular}{|c|c|}
\hline \multicolumn{2}{|c|}{ Air properties } \\
\hline Thermal conductivity & $k=2.57 \cdot 10^{-2} W(m K)$ \\
\hline Specific heat & $c_{p}=1.005 \cdot 10^{3} \mathrm{~J} /(\mathrm{kgK})$ \\
\hline Gas constant & $R=286.7 m^{2} /\left(s^{2} K\right)$ \\
\hline Temperature & $T=293.15 K$ \\
\hline Ratio of specific heats & $\gamma=1.4$ \\
\hline Fluid kinematic viscosity & $\nu_{f}=15.11 \cdot 10^{-6} \mathrm{~m}^{2} / \mathrm{s}$ \\
\hline Fluid density & $\rho_{f}=1.205 \mathrm{~kg} / \mathrm{m}^{3}$ \\
\hline \multicolumn{2}{|c|}{ Material 1: Polyurethane foam material [18] } \\
\hline Young's modulus of the frame material & $E_{s}=70 \cdot 10^{3} \mathrm{~Pa}$ \\
\hline Loss factor & $\eta_{l}=0.265$ \\
\hline Poisson ratio & $\nu=0.39$ \\
\hline Bulk density of the solid phase & $\rho_{1}=22.1 \mathrm{~kg} / \mathrm{m}^{3}$ \\
\hline Porosity & $h=0.98$ \\
\hline Viscous characteristic length & $\Lambda=1.1 \cdot 10^{-4} \mathrm{~m}$ \\
\hline Thermal characteristic length & $\Lambda^{\prime}=7.42 \cdot 10^{-4} \mathrm{~m}$ \\
\hline Static flow resistivity & $\sigma=3.75 \cdot 10^{3} \mathrm{~kg} /\left(\mathrm{m}^{3} \mathrm{~s}\right)$ \\
\hline Tortuosity & $\alpha_{\infty}=1.17$ \\
\hline \multicolumn{2}{|c|}{ Material 2: Partially reticulated polyurethane foam [43] } \\
\hline Young's modulus of the frame material & $E_{s}=8 \cdot 10^{5} \mathrm{~Pa}$ \\
\hline Loss factor & $\eta_{l}=0.265$ \\
\hline Poisson ratio & $\nu=0.4$ \\
\hline Bulk density of the solid phase & $\rho_{1}=30 \mathrm{~kg} / \mathrm{m}^{3}$ \\
\hline Porosity & $h=0.9$ \\
\hline Static flow resistivity & $\sigma=25 \cdot 10^{3} \mathrm{~kg} /\left(\mathrm{m}^{3} \mathrm{~s}\right)$ \\
\hline Tortuosity & $\alpha_{\infty}=7.8$ \\
\hline \multicolumn{2}{|c|}{ Material 3: Carpet material $[15]$} \\
\hline Young's modulus of the frame material & $E_{s}=20 \cdot 10^{3} \mathrm{~Pa}$ \\
\hline Loss factor & $\eta_{l}=0.5$ \\
\hline Poisson ratio & $\nu=0$ \\
\hline Bulk density of the solid phase & $\rho_{1}=60 \mathrm{~kg} / \mathrm{m}^{3}$ \\
\hline Porosity & $h=0.99$ \\
\hline Viscous characteristic length & $\Lambda=1.5 \cdot 10^{-4} \mathrm{~m}$ \\
\hline Thermal characteristic length & $\Lambda^{\prime}=2.2 \cdot 10^{-4} \mathrm{~m}$ \\
\hline Static flow resistivity & $\sigma=20 \cdot 10^{3} \mathrm{~kg} /\left(\mathrm{m}^{3} \mathrm{~s}\right)$ \\
\hline Tortuosity & $\alpha_{\infty}=1$ \\
\hline
\end{tabular}

Table 1: Material properties of air and two poroelastic media

forms. The FE models use a $\left(\mathbf{u}^{\mathbf{s}}, \mathbf{u}^{\mathbf{f}}\right)$-formulation. Since this paper only considers $2 \mathrm{D}$ problems, 4 dofs per node $\left(u_{x}^{s}, u_{y}^{s}, u_{x}^{f}\right.$ and $\left.u_{y}^{f}\right)$ are used. Cubic Lagrangian finite elements are used and the models are solved using a direct UMFPACK solver. Subsequent FE models are adaptively constructed, based on a L2 norm of the prediction error. The mentioned calculation times for the WBM as well 
as for the FEM include both the construction and the solution time of the system matrices since the models are frequency dependent. For these relatively small problems, also the FEM is capable of reaching higher frequencies.

\subsection{Verification case 1: rectangular poroelastic domain}

The first example consists of a simple rectangular poroelastic domain as shown in Figure 4, containing material 1 . The $\mathrm{x}$-marks indicate the locations of two postprocessing points where results will be shown. Identical mechanical boundary conditions are imposed on the four edges of the domain: no normal stress resultants on the solid nor the fluid phase and an imposed tangential stress resultant $\sigma_{s}^{s}$ of $1 \mathrm{~N} / \mathrm{m}^{2}$.

Figure 5 shows the amplitude of the obtained stress fields $\sigma_{x y}^{s}(\mathbf{r})$ and $\sigma^{f}(\mathbf{r})$. The results are obtained with the WBM using the strain formulation and a truncation factor $T=2$. The figures clearly show that the imposed boundary conditions are accurately represented.

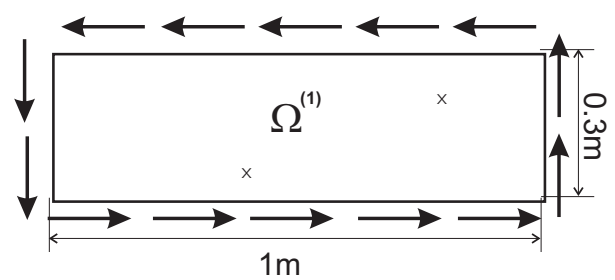

Figure 4: Problem description of the rectangular poroelastic domain, $\mathrm{x}$ postprocessing points

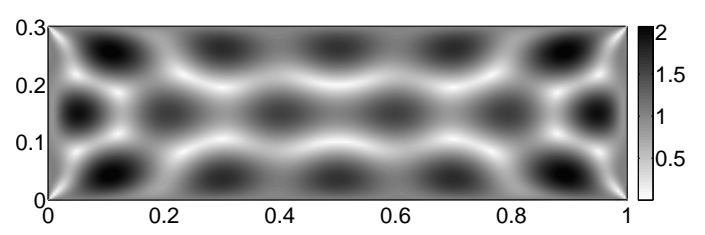

(a) $\sigma_{x y}^{s}(\mathbf{r})\left[N / m^{2}\right]$

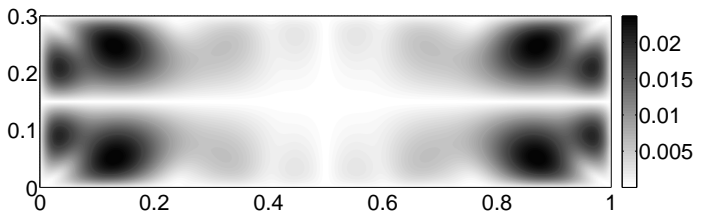

(b) $\sigma^{f}(\mathbf{r})\left[N / m^{2}\right]$

Figure 5: Amplitude of some stress fields at $150 \mathrm{~Hz}$

To verify that the WBM is capable of representing the same results as the FEM, frequency response functions (FRFs) are calculated with both the strain and the potential formulation. Figure 6 shows the FRFs of the fluid displacement in the x-direction $u_{x}^{f}(\mathbf{r})$ and the shear stress $\sigma_{x y}^{s}(\mathbf{r})$ 
obtained in two different points in the problem domain. For the FEM reference, 13 adaptive h-refinements are performed to obtain accurate results. These mesh refinements are frequencydependent, but for all frequency lines approximately 780,000 dofs are used. These figures show no visual difference between the obtained results for both WBM formulations and the results obtained with the FEM.

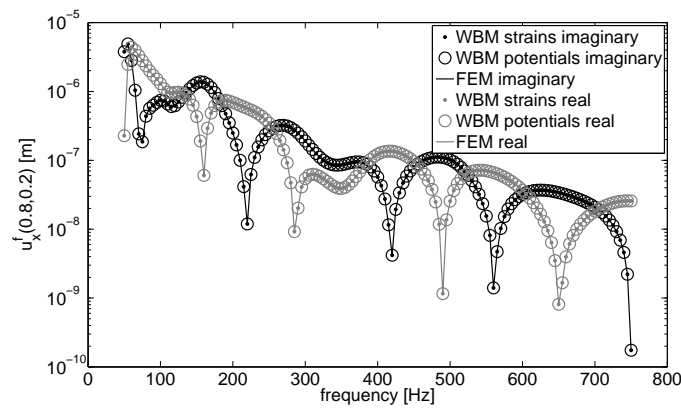

(a) $u_{x}^{f}(\mathbf{r})$

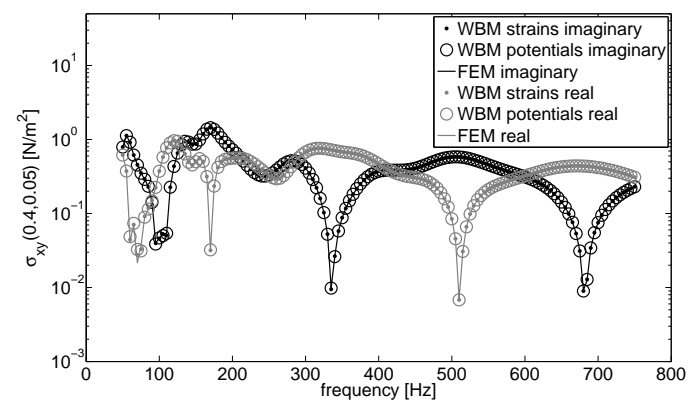

(b) $\sigma_{x y}^{s}(\mathbf{r})$

Figure 6: FRFs of respectively $u_{x}^{f}(\mathbf{r})$ and $\sigma_{x y}^{s}(\mathbf{r})$ in two different response points calculated with the WBM and the FEM

Convergence curves at $200 \mathrm{~Hz}$ and $400 \mathrm{~Hz}$ are calculated to demonstrate the beneficial convergence rate of the WBM as compared to the FEM. For both frequencies the FE calculations start from a model consisting of 10 finite elements (232 dofs). This model is refined 15 times, using an adaptive h-refinement based on the L2-error in the domains and the details of the different models are summarised in Table 2. This approach has been chosen to obtain a high accuracy and also a higher convergence rate as compared to non-adaptively refined meshes. The finest models are used as reference. The reference models at 200 and $400 \mathrm{~Hz}$ have nearly the same number of dofs. This is due to memory restrictions of the available hardware. As a result, the reference at $400 \mathrm{~Hz}$ is not as accuarate as the one at $200 \mathrm{~Hz}$. Also different WB models are constructed. For both the strain and the potential formulation, the same numbers of wave functions are applied. For the first WB model, 60 wave functions of each kind are used, both at $200 \mathrm{~Hz}$ as well as at $400 \mathrm{~Hz}$. These numbers are increased such that the finest WB model contains 740 wave functions of each kind at $200 \mathrm{~Hz}$ and 1,112 at $400 \mathrm{~Hz}$. To compare the convergence rate of the different methods, the relative prediction error $|\epsilon|$ of a variable $a$ is calculated as a function of CPU time. This prediction error is averaged 


\begin{tabular}{|c|c|r|r|r|r|}
\cline { 3 - 6 } \multicolumn{2}{c|}{} & \multicolumn{2}{c|}{$200 \mathrm{~Hz}$} & \multicolumn{2}{c|}{$400 \mathrm{~Hz}$} \\
\hline $\mathrm{nr}$ & $\sharp$ refinements & dofs & cputime $[\mathrm{s}]$ & dofs & cputime [s] \\
\hline 1 & 0 & 232 & 1.10 & 232 & 1.34 \\
\hline 2 & 1 & 484 & 1.17 & 484 & 1.74 \\
\hline 3 & 2 & 964 & 1.58 & 964 & 1.88 \\
\hline 4 & 3 & 1,828 & 2.42 & 1,852 & 1.84 \\
\hline 5 & 4 & 3,652 & 2.29 & 3,364 & 2.02 \\
\hline 6 & 5 & 7,084 & 3.44 & 6,412 & 2.78 \\
\hline 7 & 6 & 13,252 & 5.21 & 12,340 & 4.35 \\
\hline 8 & 7 & 24,892 & 7.72 & 22,636 & 7.34 \\
\hline 9 & 8 & 43,636 & 14.18 & 41,932 & 13.49 \\
\hline 10 & 9 & 77,332 & 25.80 & 72,916 & 24.40 \\
\hline 11 & 10 & 133,276 & 51.46 & 122,788 & 45.92 \\
\hline 12 & 11 & 245,188 & 94.96 & 229,180 & 86.49 \\
\hline 13 & 12 & 454,324 & 202.89 & 426,268 & 188.19 \\
\hline 14 & 13 & 791,860 & 398.69 & 778,396 & 383.78 \\
\hline 15 & 14 & $1,430,956$ & 850.71 & $1,409,380$ & 829.40 \\
\hline 16 & 15 & $2,286,388$ & $1,925.58$ & $2,277,124$ & $1,908.79$ \\
\hline
\end{tabular}

Table 2: FEM model data of the rectangular problem domain

over $N$ response points in the poroelastic domain:

$$
|\epsilon|=\frac{1}{N} \sum_{j=1}^{N} \epsilon_{j} \quad \text { with } \quad \epsilon_{j}=\frac{\left\|a\left(\mathbf{r}_{\mathbf{j}}\right)-a^{r e f}\left(\mathbf{r}_{\mathbf{j}}\right)\right\|}{\left\|a^{r e f}\left(\mathbf{r}_{\mathbf{j}}\right)\right\|}
$$

For this example, $N=20$ equally distributed response points are considered. Figures 7 and 8 show convergence curves of $u_{y}^{f}(\mathbf{r})$ and $\sigma_{x y}^{s}(\mathbf{r})$ at respectively 200 and $400 \mathrm{~Hz}$. The WB models obtain the same accuracy as the FE reference but require much less calculation time. At $400 \mathrm{~Hz}$, the better convergence is more pronounced, indicating the potential of the proposed method to tackle problems at higher frequencies. The relative errors are, in these cases, constrained by the obtainable accuracy of the FE reference model. The behaviour of the convergence curves obtained by the strain and the potential formulation is similar. The differences are due to slightly different condition numbers of the system matrices.

Since the convergence inside the problem domain depends on the convergence of the boundary residuals, these convergences are shown in Figure 9(a). It shows the absolute value of the residuals on the lower boundary, indicated by $\Gamma_{1}$, of the problem domain, averaged over 19 evenly distributed points, calculated with the strains and the potential formulation at $400 \mathrm{~Hz}$. The results converge, but somewhat slower as compared with the results within the domain. Again, the differences between 


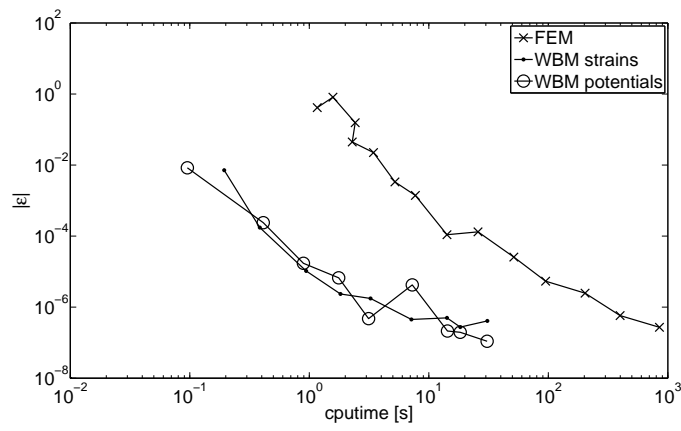

(a) $u_{y}^{f}(\mathbf{r})$

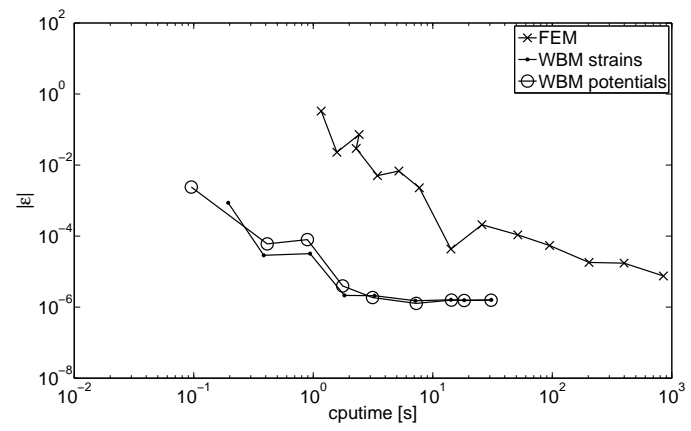

(b) $\sigma_{x y}^{s}(\mathbf{r})$

Figure 7: Convergence curves at $200 \mathrm{~Hz}$, calculated with the FEM and the WBM, applying the strain and potential formulation

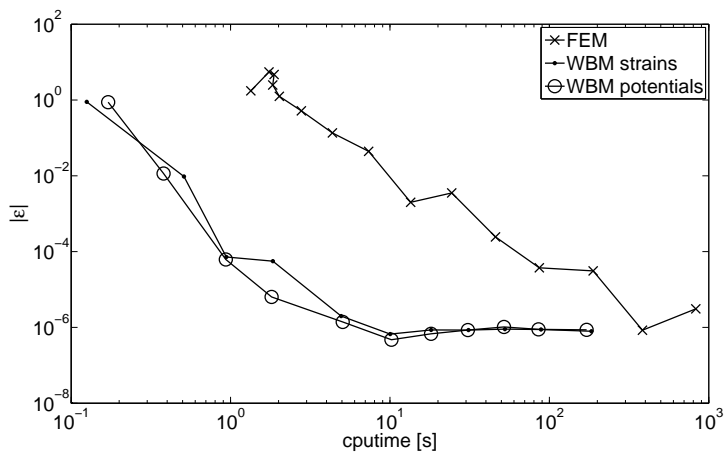

(a) $u_{y}^{f}(\mathbf{r})$

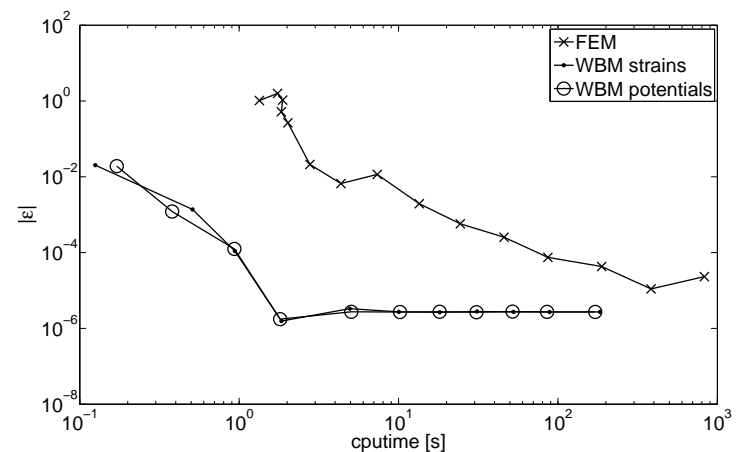

(b) $\sigma_{x y}^{s}(\mathbf{r})$

Figure 8: Convergence curves at $400 \mathrm{~Hz}$ calculated with the FEM and the WBM, applying the strain and potential formulation

the strain and the potential formulation are small. Figure 9 shows the residuals of the imposed shear stress condition on the four boundaries, numbered clockwise, of the imposed shear stress. Similar convergence curves are obtained for the four boundaries.

To validate the coupling conditions between two poroelastic domains as defined in equation (10), the rectangular problem domain of Figure 4 is divided into two subdomains of different sizes as shown in Figure 10. Figure 11 shows contour plots of the displacement field $u_{x}^{f}(\mathbf{r})$ and the stress field $\sigma_{y}^{s}(\mathbf{r})$ calculated with the WB strain formulation. Again a truncation factor $T=2$ is applied. No discontinuities can be seen at the interface between the two subdomains $\Omega^{(1)}$ and $\Omega^{(2)}$, 


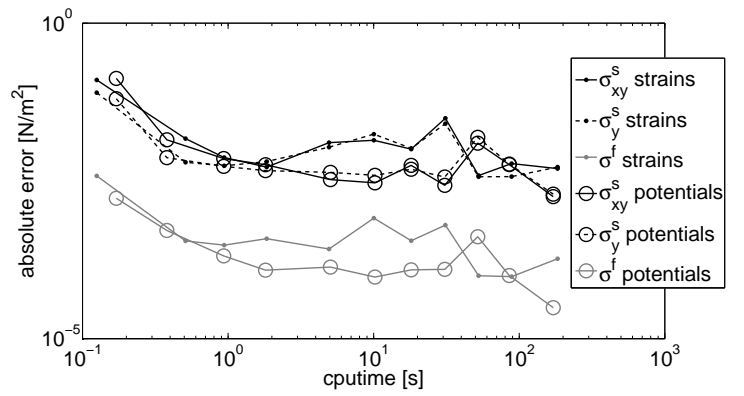

(a) Absolute value of the averaged error of the imposed values on $\Gamma_{1}$

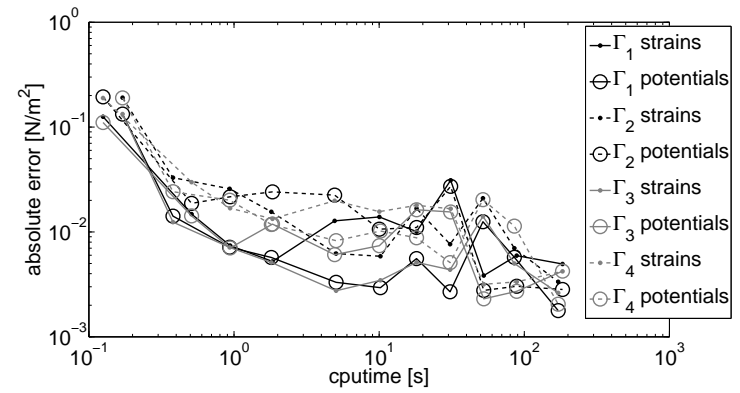

(b) Absolute value of the averaged error on $\sigma_{n s}^{s}$ on the four boundaries

Figure 9: Convergence curves of the residuals at $200 \mathrm{~Hz}$, calculated with the WBM, applying the strain and potential formulation

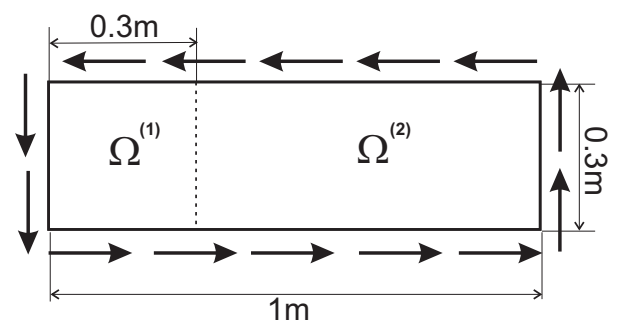

Figure 10: Problem description of the rectangular poroelastic domain

indicating that the coupling between the two subdomains is accurately represented.

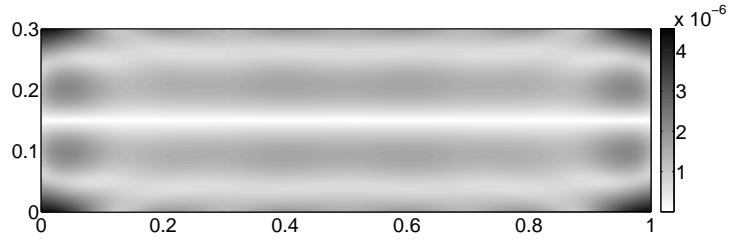

(a) $u_{x}^{f}(\mathbf{r})[m]$

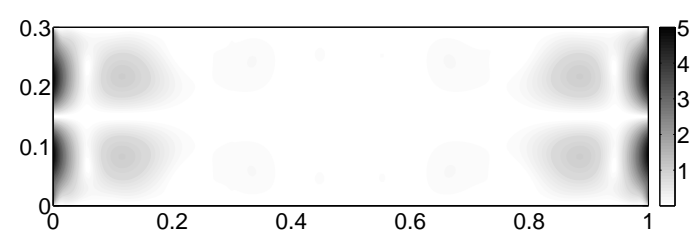

(b) $\sigma_{y}^{s}(\mathbf{r})\left[N / m^{2}\right]$

Figure 11: Contour plots of different fields in the poroelastic rectangle consisting of two WB subdomains

Convergence curves are shown in Figures 12 and 13. These figures compare the convergence curve of the WBM with the strain formulation for the geometry of Figure 4 and Figure 10 at $200 \mathrm{~Hz}$ and $400 \mathrm{~Hz}$ respectively. The numbers of wave functions of each type are increased from 12 to 340 for subdomain $\Omega^{(1)}$ and from 24 to 568 for subdomain $\Omega^{(2)}$ at $200 \mathrm{~Hz}$, and from 28 to 452 


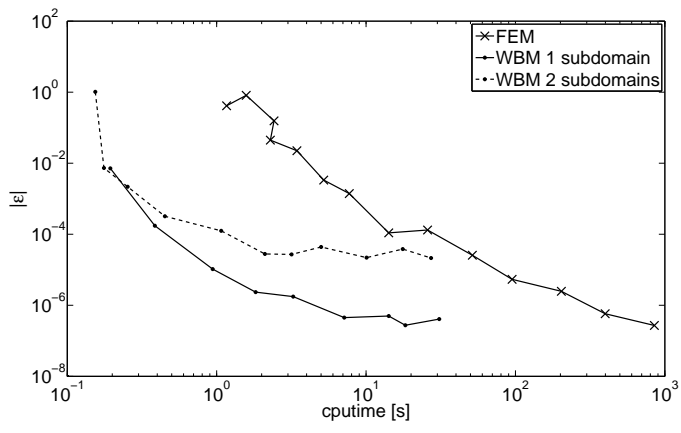

(a) $u_{y}^{f}(\mathbf{r})$

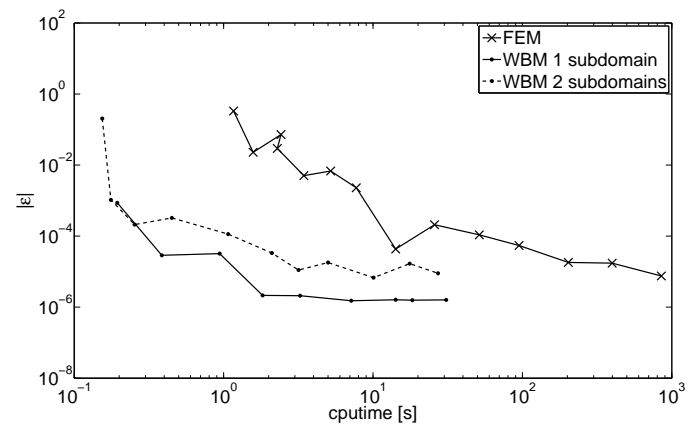

(b) $\sigma_{x y}^{s}(\mathbf{r})$

Figure 12: Convergence curves at 200Hz, comparing the FEM and the WBM with one and two subdomains, applying the strain formulation

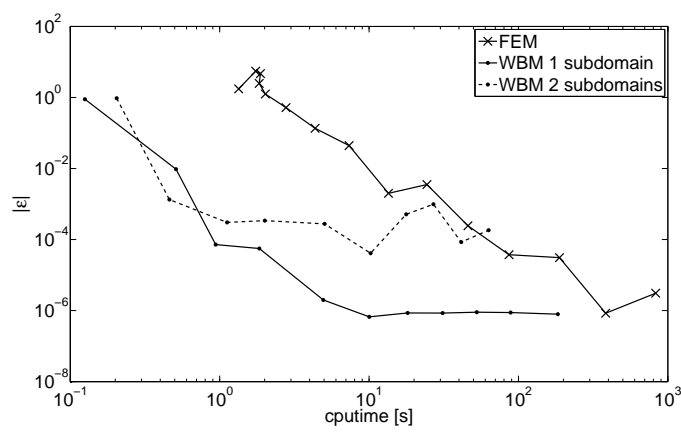

(a) $u_{y}^{f}(\mathbf{r})$

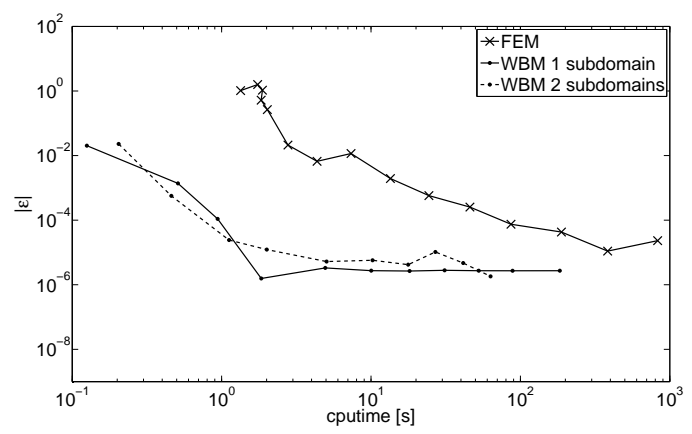

(b) $\sigma_{x y}^{s}(\mathbf{r})$

Figure 13: Convergence curves at $400 \mathrm{~Hz}$, comparing the FEM and the WBM with one and two subdomains, applying the strain formulation

for subdomain $\Omega^{(1)}$ and from 48 to 756 for subdomain $\Omega^{(2)}$ at $400 \mathrm{~Hz}$. As can be seen from those figures, the WBM with two subdomains obtains engineering accuracy very fast, but stagnates at a lower accuracy as compared to the WBM with one subdomain. This is due to the worse condition number of the system of equations. Nevertheless, regardless of the considered frequency a very good accuracy is obtained.

\subsection{Verification case 2: quadrangular poroelastic domain}

The second verification example utilises the WBM for a non-rectangular problem domain as shown in Figure 14 and considers various boundary conditions and various materials, respectively. 


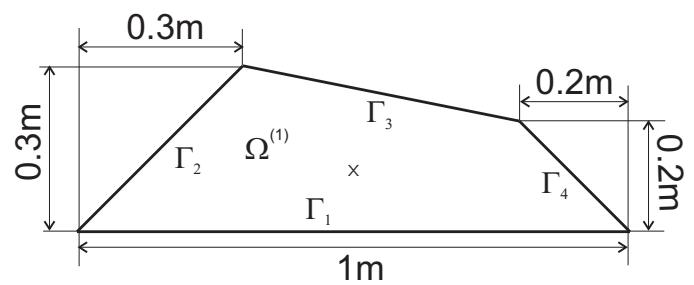

Figure 14: Problem description of the quadrangular poroelastic domain, $\mathrm{x}$ postprocessing point

\begin{tabular}{c|c|c|c} 
Set & Type & Imposed values & Boundary \\
\hline \multirow{2}{*}{1} & mechanical boundary conditions & $\sigma_{n}^{s}(\mathbf{r})=\sigma_{s}^{s}(\mathbf{r})=0 N / m^{2}, \sigma^{f}(\mathbf{r})=1 N / m^{2}$ & $\Gamma_{2}, \Gamma_{3}, \Gamma_{4}$ \\
& kinematic boundary conditions & $u_{n}^{s}(\mathbf{r})=u_{s}^{s}(\mathbf{r})=u_{n}^{f}(\mathbf{r})=0 m$ & $\Gamma_{1}$, \\
\hline \multirow{3}{*}{2} & mechanical boundary conditions & $\sigma_{n}^{s}(\mathbf{r})=1 N / m^{2}, \sigma_{s}^{s}(\mathbf{r})=\sigma^{f}(\mathbf{r})=0 N / m^{2}$ & $\Gamma_{3}$ \\
& mechanical boundary conditions & $\sigma_{n}^{s}(\mathbf{r})=\sigma_{s}^{s}(\mathbf{r})=\sigma^{f}(\mathbf{r})=0 N / m^{2}$ & $\Gamma_{2}, \Gamma_{4}$ \\
& mixed boundary conditions & $u_{n}^{s}(\mathbf{r})=\sigma_{s}^{s}(\mathbf{r})=u_{n}^{f}(\mathbf{r})=0$ & $\Gamma_{1}$ \\
\hline
\end{tabular}

Table 3: Applied sets of boundary conditions

Firstly, two sets of boundary conditions are evaluated and compared with respect to accuracy. Next, two different materials are compared. All WB calculations are compared with FE reference results.

\subsubsection{Different sets of boundary conditions}

Two different sets of boundary conditions are evaluated for the WB approach. Table 3 shows both sets. The first set contains one fixed boundary and on the other three boundaries a stress resultant is imposed on the fluid phase. The second set contains one sliding edge, two free boundaries and on one boundary a normal stress resultant on the solid phase is applied. In this way, all types of possible boundary conditions are covered. For both configurations, material 1 is applied.

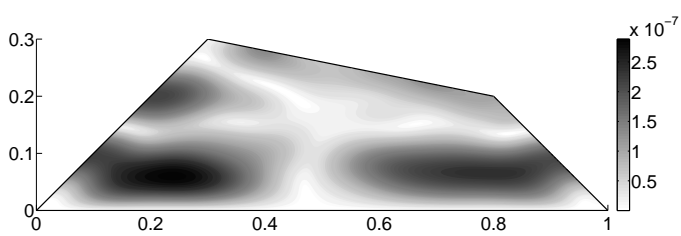

(a) Boundary conditions set 1

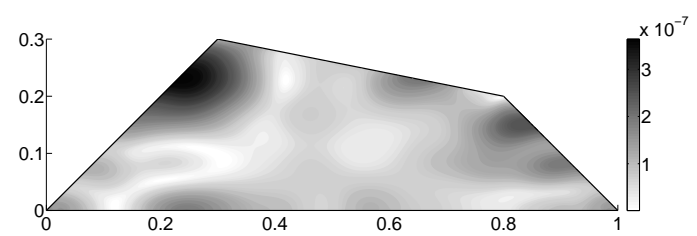

(b) Boundary conditions set 2

Figure 15: Contour plots of the amplitude of $u_{x}^{s}(\mathbf{r})[\mathrm{m}]$ for both sets of boundary conditions at $220 \mathrm{~Hz}$, calculated with the WBM strain formulation 


\begin{tabular}{|c|c|c|c|c|}
\cline { 2 - 5 } \multicolumn{1}{c|}{} & \multicolumn{2}{c|}{$200 \mathrm{~Hz}$} & \multicolumn{2}{c|}{$400 \mathrm{~Hz}$} \\
\hline boundary conditions & dofs & cputime [s] & dofs & cputime [s] \\
\hline Set 1 & $1,751,416$ & 1,128 & $2,237,548$ & 1,711 \\
\hline Set 2 & $1,682,860$ & 1,092 & $1,568,462$ & 1,018 \\
\hline
\end{tabular}

Table 4: FEM reference data for the quadrangular problem domain considering 2 sets of boundary conditions, containing material 1

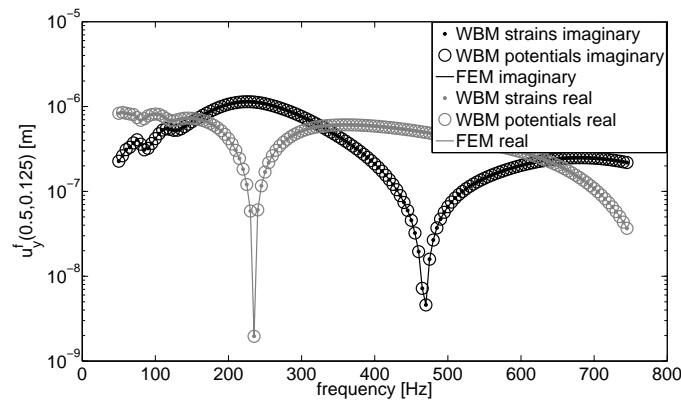

(a) Boundary conditions set 1

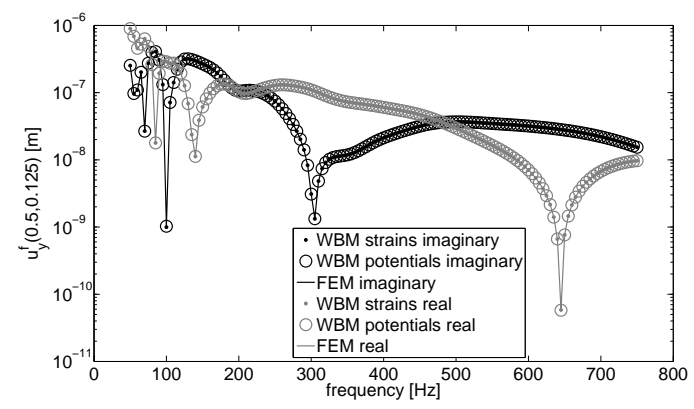

(b) Boundary conditions set 2

Figure 16: FRFs of the amplitude of $u_{y}^{f}(0.5,0.125)[\mathrm{m}]$ for both sets of boundary conditions, calculated with the WBM strain and potential formulation and the FEM

Figure 15 contains contour plots of the amplitude of the x-component of the solid phase at $220 \mathrm{~Hz}$ for both sets of boundary conditions using the WB strain formulation and a truncation factor $T=2$. For set 1 , the imposed boundary conditions on $\Gamma_{1}$ are clearly fulfilled.

Figure 16 shows FRFs of the amplitude of $u_{y}^{f}(\mathbf{r})$ calculated in the response point with coordinates $(0.5,0.125)$ for both problem configurations with the two WB formulations and the FEM. For the FEM reference, 13 adaptive refinements are performed for each frequency, resulting in approximately 520,000dofs. A very good agreement is obtained for the considered frequency range and both configurations.

Convergence curves are calculated at $200 \mathrm{~Hz}$ and at $400 \mathrm{~Hz}$ for both sets of boundary conditions applying both WB formulations as well as the FEM. 15 adaptive refinements are performed at both frequencies and for the two sets of applied boundary conditions to obtain very accurate FE reference models. Table 4 contains the details for the 4 obtained reference models.

For both sets of boundary conditions, the number of each type of wave functions is increased from 28 to 496 at $200 \mathrm{~Hz}$ and from 60 to 492 at $500 \mathrm{~Hz}$ for both the WB strain and potential formulations. 


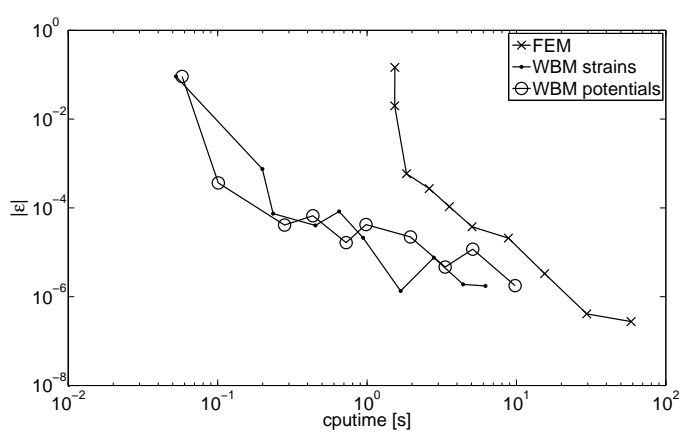

(a) $u_{x}^{s}(\mathbf{r})$, boundary conditions set 1

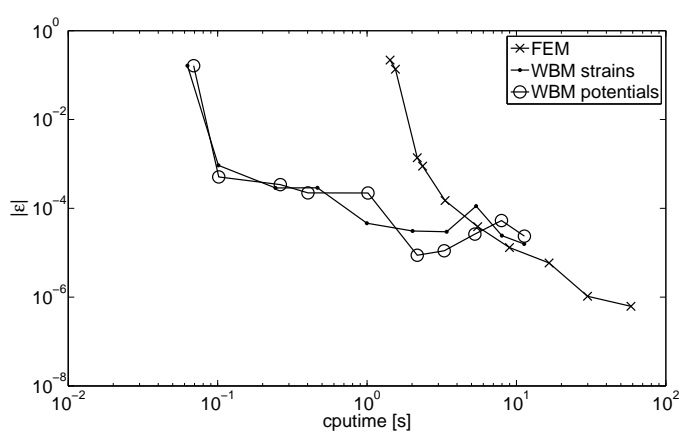

(c) $u_{x}^{s}(\mathbf{r})$, boundary conditions set 2

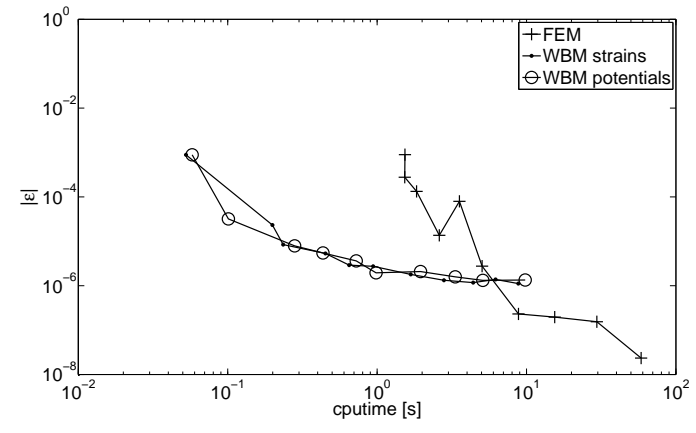

(b) $\sigma^{f}(\mathbf{r})$, boundary conditions set 1

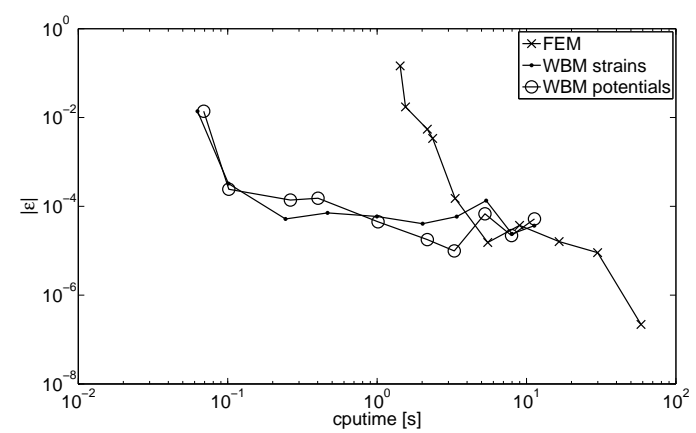

(d) $\sigma^{f}(\mathbf{r})$, boundary conditions set 2

Figure 17: Convergence curves of $u_{x}^{s}(\mathbf{r})$ and $\sigma^{f}(\mathbf{r})$ at $200 \mathrm{~Hz}$ for both sets of boundary conditions calculated with the WB strain and potential formulation and the FEM

Figure 17 and Figure 18 shows the convergence curves obtained with the WBM and the adaptive FEM at respectively $200 \mathrm{~Hz}$ and $400 \mathrm{~Hz}$ for both sets of boundary conditions. The relative prediction error, in equation (24), is calculated for $u_{x}^{s}(\mathbf{r})$ and $\sigma^{f}(\mathbf{r})$ and is averaged over $N=58$ response points distributed over the problem domain. The figures show that the WBM converges very fast to engineering precision but stagnates at a certain accuracy. This is due to the condition number of the system matrix, which becomes higher when adding more wave functions with little influence on the field. Nevertheless, engineering accuracy is obtained at least ten times faster than the FEM. A similar convergence is obtained for both the strain and potential formulations. The applied boundary conditions have a small influence on the obtained accuracy. 


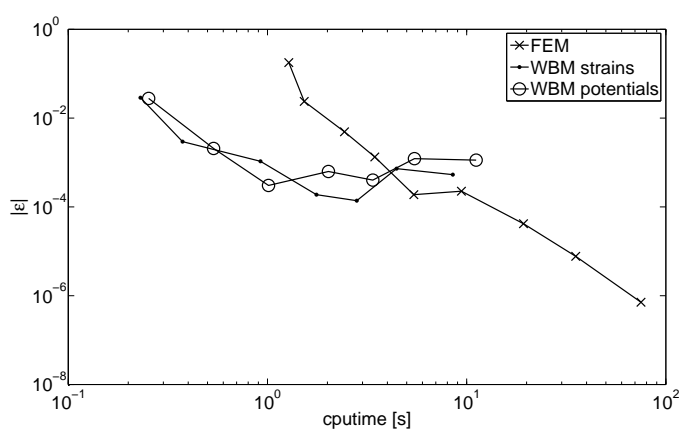

(a) $u_{x}^{s}(\mathbf{r})$, boundary conditions set 1

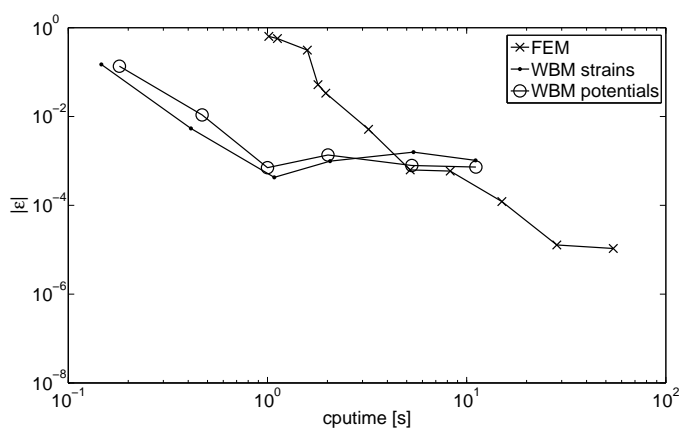

(c) $u_{x}^{s}(\mathbf{r})$, boundary conditions set 2

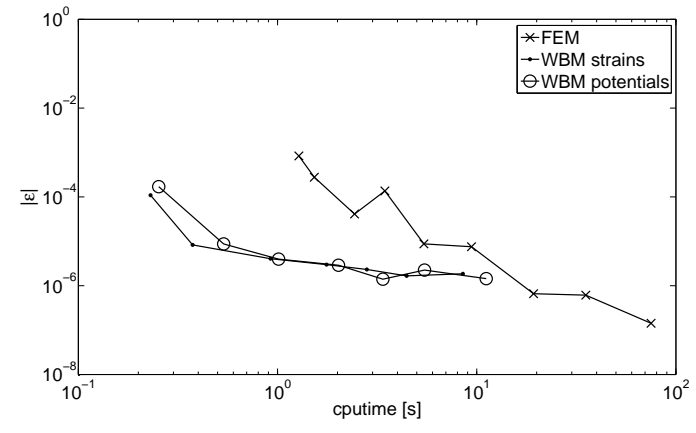

(b) $\sigma^{f}(\mathbf{r})$, boundary conditions set 1

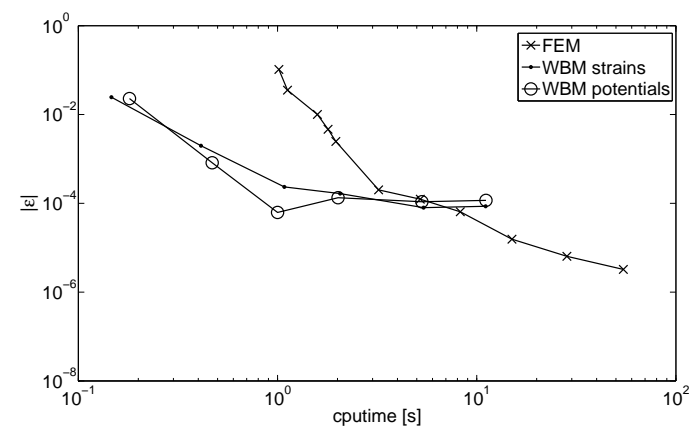

(d) $\sigma^{f}(\mathbf{r})$, boundary conditions set 2

Figure 18: Convergence curves of $u_{x}^{s}(\mathbf{r})$ and $\sigma^{f}(\mathbf{r})$ at $400 \mathrm{~Hz}$ for both sets of boundary conditions calculated with the WB strain and potential formulation and the FEM

\subsubsection{Different materials}

Finally, the proposed method is also applied for another poroelastic material. This section combines the second material of Table 1 and the first set of boundary conditions of Table 3. To indicate the influence of the chosen material, Figure 19 shows contour plots of the amplitude of $\sigma_{y}^{s}(\mathbf{r})$ at $350 \mathrm{~Hz}$ for both material 1 and 2 applying the same boundary conditions. A different behaviour is obtained.

Figure 20 shows the FRFs of $u_{y}^{f}(0.5,0.125)$ obtained with the WBM and the FEM. For the WBM a truncation factor of $T=2$ is applied and 13 adaptive refinements are performed to obtain the FE reference results. Regardless of the formulation, the WBM is capable to represent the same results.

Also convergence curves are calculated at both $200 \mathrm{~Hz}$ and 400Hz. 15 adaptive refinements are 


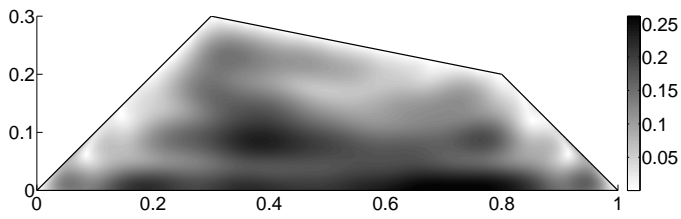

(a) Material 1

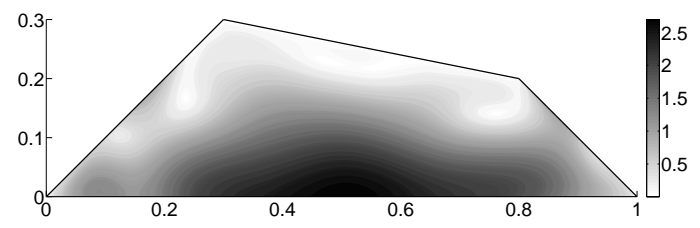

(b) Material 2

Figure 19: Contour plots of the amplitude of $\sigma_{y}^{s}(\mathbf{r})\left[\mathrm{N} / \mathrm{m}^{2}\right]$ for both sets of materials at $350 \mathrm{~Hz}$, calculated with the WBM strains formulation

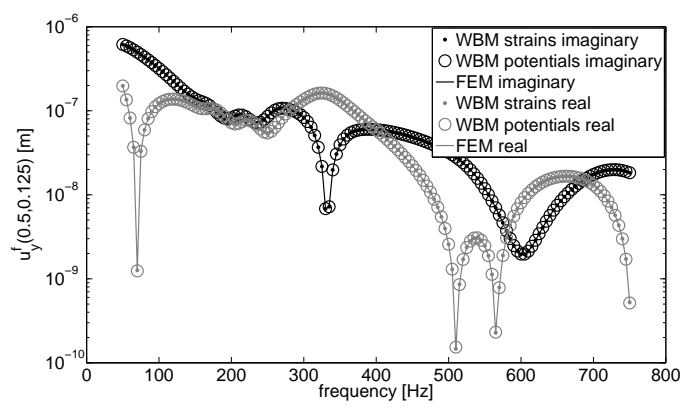

Figure 20: FRF of $u_{y}^{f}(0.5,0.125)$ calculated with the WBM, both strains and potentials formulation and the FEM

performed to obtain the FEM references as given in Table 5. The number of wave functions of each type is increased from 12 to 216 at $200 \mathrm{~Hz}$ and from 24 to 356 at $400 \mathrm{~Hz}$. The results, shown in Figures 21 and 22, are similar as for the previous examples, indicating that, irrespective of the type of material, the WBM converges to engineering precision in a very small calculation time when compared with the FEM.

\subsection{Verification case 3: multilayer example}

The last verification example verifies the ability of the WBM to predict the behaviour inside a multilayer consisting of 3 layers of poroelastic materials. The lay-out is shown in Figure 23. The top and the bottom layer contain material 1, the middle layer consists of material 3 of Table

\begin{tabular}{|c|c|c|c|c|}
\cline { 2 - 5 } \multicolumn{1}{c|}{} & \multicolumn{2}{c|}{$200 \mathrm{~Hz}$} & \multicolumn{2}{c|}{$400 \mathrm{~Hz}$} \\
\hline boundary conditions & dofs & cputime $[\mathrm{s}]$ & dofs & cputime $[\mathrm{s}]$ \\
\hline Set 1 & $1,509,520$ & 1,003 & $1,616,464$ & 1,045 \\
\hline
\end{tabular}

Table 5: FEM reference data for the quadrangular problem containing material 2 and applying set 1 of boundary conditions 


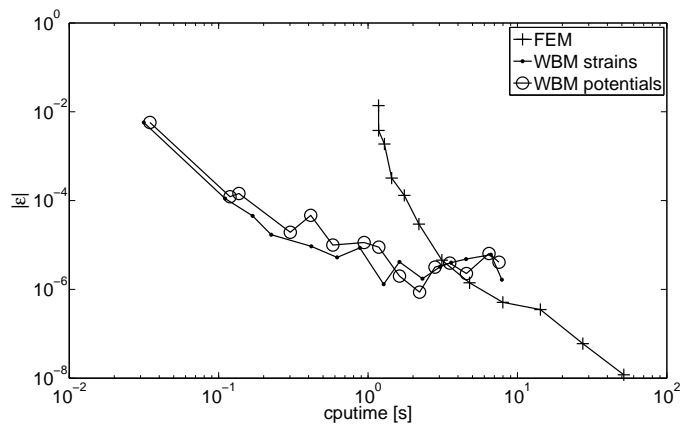

(a) $u_{x}^{s}(\mathbf{r})[m]$

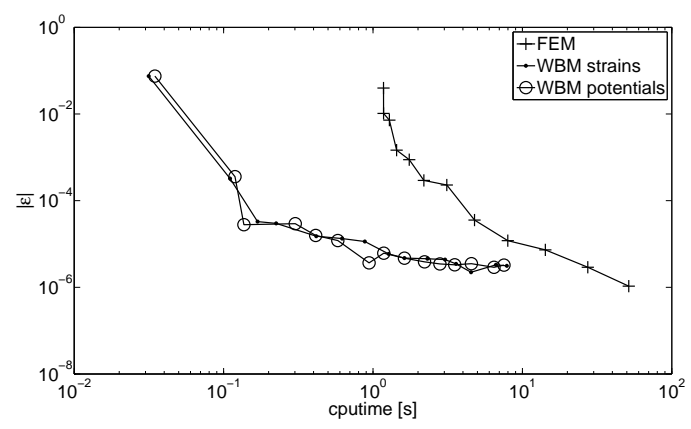

(b) $\sigma^{f}(\mathbf{r})\left[N / m^{2}\right]$

Figure 21: Convergence curves of $u_{x}^{s}(\mathbf{r})$ and $\sigma^{f}(\mathbf{r})$ at $200 \mathrm{~Hz}$ for material 2 and set 1 of boundary conditions calculated with the WB strain and potential formulations and the FEM

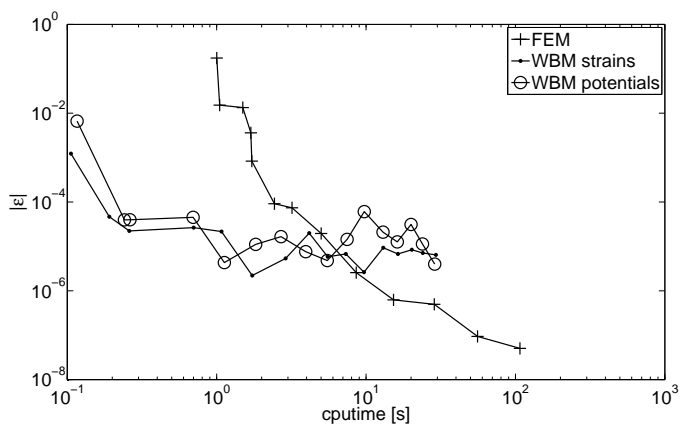

(a) $u_{x}^{s}(\mathbf{r})[m]$

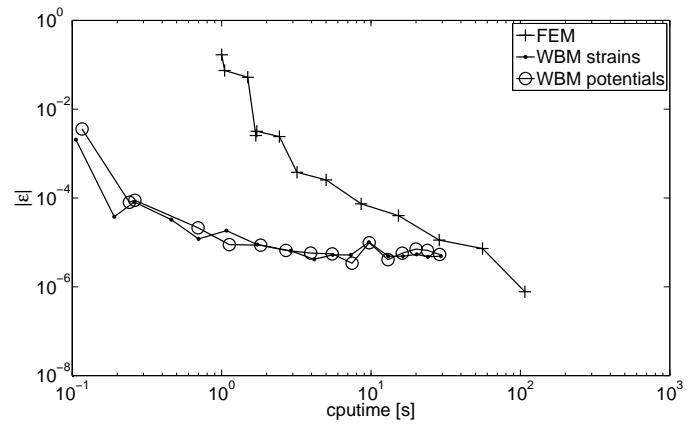

(b) $\sigma^{f}(\mathbf{r})\left[N / m^{2}\right]$

Figure 22: Convergence curves of $u_{x}^{s}(\mathbf{r})$ and $\sigma^{f}(\mathbf{r})$ at $400 \mathrm{~Hz}$ for material 2 and set 1 of boundary conditions calculated with the WB strain and potential formulations and the FEM

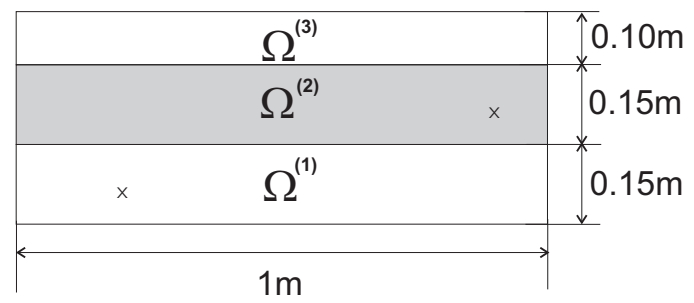

Figure 23: Problem description of a multilayer, $\mathrm{x}$ postprocessing points

1. On all boundaries, except for the top one, sliding edge conditions (9)[15] are imposed. On the top layer an acoustic pressure of the form $p(x)=x^{3}-2 x^{2}+1 N / m^{2}$ excites the system. A 
distributed pressure of an arbitrary shape is chosen here since a constant pressure would only excite the longitudinal wave types. This leads to the following mechanical boundary conditions on the top layer: $\sigma^{f(3)}=-h^{(3)} p^{a}, \sigma_{y}^{s(3)}=-\left(1-h^{(3)}\right) p^{a}$ and $\sigma_{x y}^{s}=0 N / m^{2}$. On the interfaces between different layers, coupling conditions (10) are imposed with porosities according to the material within each subdomain.

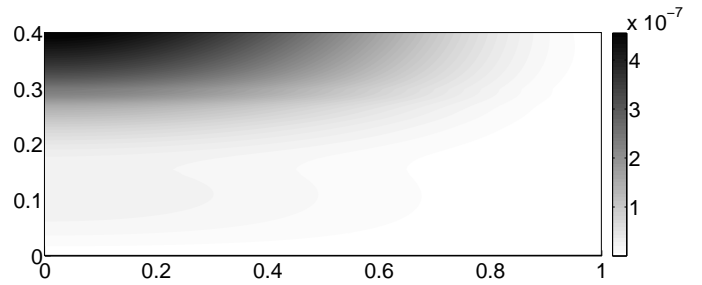

(a) $h^{(\alpha)}\left(u_{y}^{f(\alpha)}(\mathbf{r})-u_{y}^{s(\alpha)}(\mathbf{r})\right)[m]$

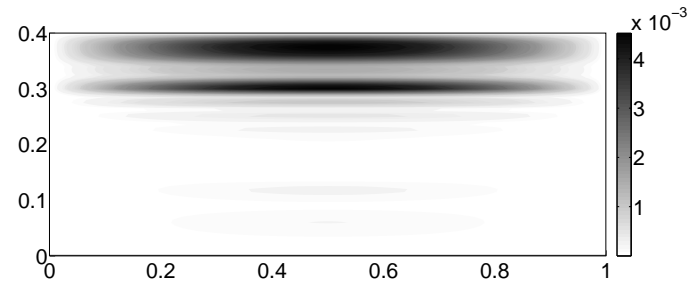

(b) $\sigma^{s(\alpha)}(\mathbf{r})\left[N / m^{2}\right]$

Figure 24: Contour plots of different continuous fields in the multilayer, calculated at $600 \mathrm{~Hz}$ calculated with the WBM potentials formulation

Figure 24(a) shows the absolute value of the relative mass flux and Figure 24(b), shows the absolute value of the shear stresses in the three layers calculated with the WBM at $600 \mathrm{~Hz}$ using the potential formulation. The WB model contains 408 wave functions of each kind in subdomain $\Omega^{(1)}$ and $\Omega^{(2)}$ and 388 wave functions of each type in subdomain $\Omega^{(3)}$. Continuity is clearly obtained across the interfaces. In order to verify the coupling between the domains, Figure 25 shows the $x$ components of the displacement of the solid phases on the interface between $\Omega^{(1)}$ and $\Omega^{(2)}$. Visually, there are no differences. The absolute error is very small, indicating a good coupling between the quantities in both subdomains.
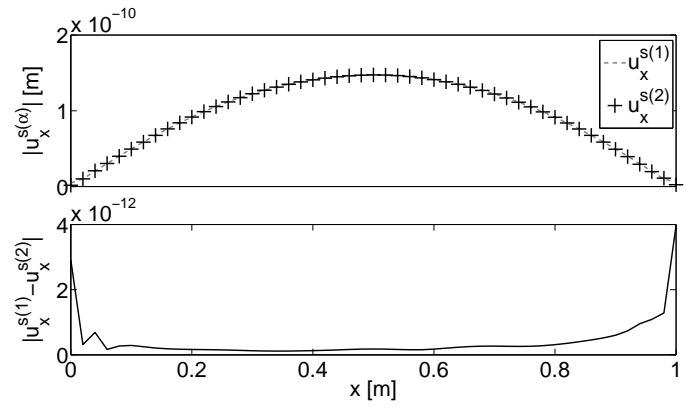

Figure 25: Evaluation of the coupling conditions on $u_{x}^{s}$ between subdoman $\Omega^{(1)}$ and $\Omega^{(2)}$ 


\begin{tabular}{|c|c|c|c|}
\hline \multicolumn{2}{|c|}{$200 \mathrm{~Hz}$} & \multicolumn{2}{c|}{$400 \mathrm{~Hz}$} \\
\hline dofs & cputime $[\mathrm{s}]$ & dofs & cputime $[\mathrm{s}]$ \\
\hline $1,285,260$ & 779 & $1,157,736$ & 691 \\
\hline
\end{tabular}

Table 6: FEM reference data for the Multilayer problem

Figure 26 compares FRFs of respectively $u_{y}^{s}$ and $\sigma^{f}$ obtained in two different points in subdomains $\Omega^{(1)}$ and $\Omega^{(2)}$ calculated with the FEM and the WBM. The FE results are obtained applying 6 adaptive refinements, leading to 330.000 dofs per frequency line. The results of both methods coincide, indicating that a good accuracy is obtained.

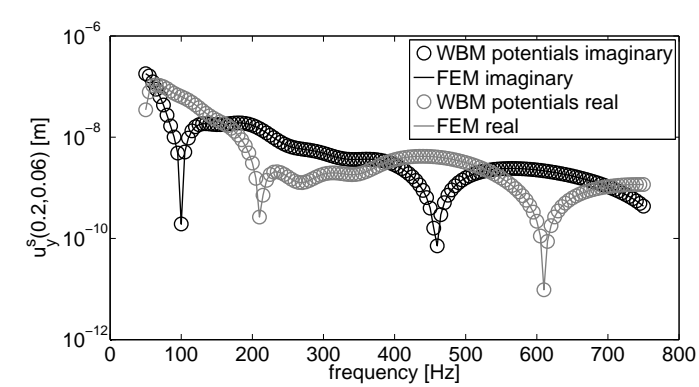

(a) $u_{y}^{s(1)}(\mathbf{r})[m]$

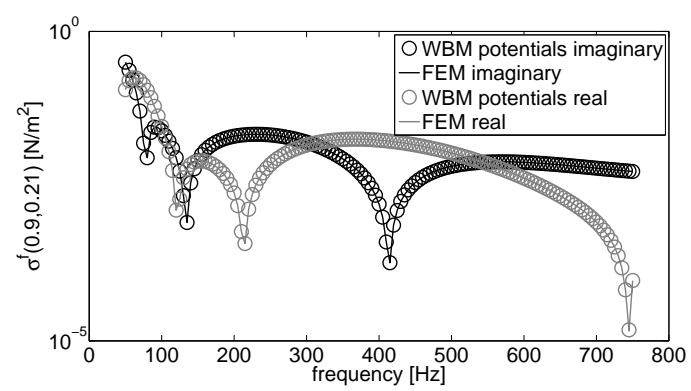

(b) $\sigma^{f(2)}(\mathbf{r})\left[N / m^{2}\right]$

Figure 26: Frequency response functions of two different field variables obtained in two different response points in subdomains $\Omega^{(1)}$ and $\Omega^{(2)}$ applying both the FEM and the WB potentials formulation.

Convergence curves have been calculated both at $200 \mathrm{~Hz}$ and $400 \mathrm{~Hz}$ with the FEM and the WBM potentials formulation. 8 adaptive refinements are performed on the first FE mesh, consisting of 5688 dofs. The finest model is used as a reference. Table 6 contains the FE reference data. In the WB model, the number of wave functions for each type is gradually increased from 24 till 544 for subdomain 1 and 2 and from 24 till 520 for subdomain 3 at $200 \mathrm{~Hz}$ and from 52 till 1084 for subdoamin 1 and 2 and from 52 till 1036 for subdomain 3 at 400Hz. The relative prediction error (24) is calculated for $u_{x}^{f}$ and $\sigma_{x y}^{s}$ in each subdomain and is averaged over 36 equally distributed response points. The convergence curves at $200 \mathrm{~Hz}$ and $400 \mathrm{~Hz}$ are shown in Figure 27 and Figure 28 respectively. Although different materials are combined containing waves with totally different wavelengths, an excellent accuracy is obtained with the WB approach. 


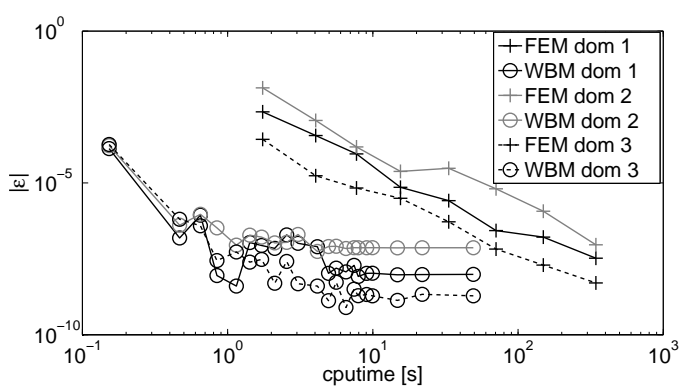

(a) $u_{x}^{f}(\mathbf{r})[m]$

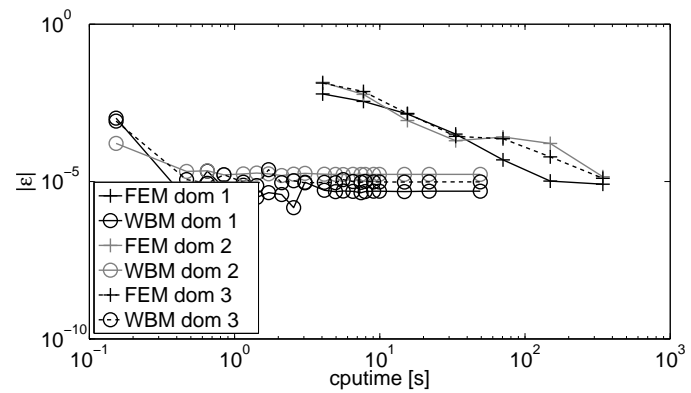

(b) $\sigma_{x y}^{s}(\mathbf{r})\left[N / m^{2}\right]$

Figure 27: Convergence curves of $u_{x}^{f}(\mathbf{r})$ and $\sigma_{x y}^{s}(\mathbf{r})$ at $200 \mathrm{~Hz}$ in each of the three subdomains, calculated with the WB potential formulation and the FEM

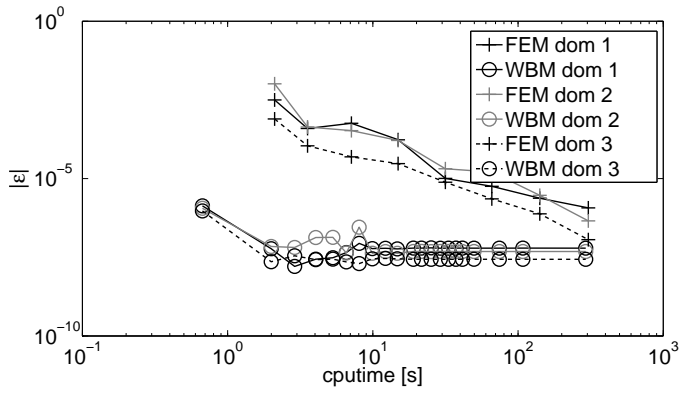

(a) $u_{x}^{f}(\mathbf{r})[m]$

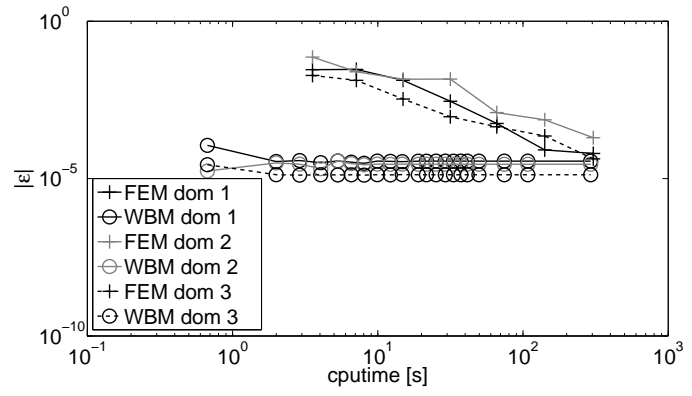

(b) $\sigma_{x y}^{s}(\mathbf{r})\left[N / m^{2}\right]$

Figure 28: Convergence curves of $u_{x}^{f}(\mathbf{r})$ and $\sigma_{x y}^{s}(\mathbf{r})$ at $400 \mathrm{~Hz}$ in each of the three subdomains, calculated with the WB potential formulation and the FEM

\section{Conclusions}

This paper presents a Wave Based prediction technique to solve the 2D Biot equations for poroelastic materials. The basic principles of the WBM are discussed and are applied to the Biot equations. The equations can be decoupled into three Helmholtz equations in two different ways: using a strain or a potential formulation. Exact solutions of these three Helmholtz equations are used in an expansion set to approximate the field variables. In this way, the physics of the three propagating wave types in a porous material are explicitly taken into account. The application of exact solutions leads to a lower number of degrees of freedom and a faster solution time. Three verification cases show the potential of the proposed method and compare the convergence of the 
method to cubic adaptive Finite Element models for geometrically simple problem domains. The WBM has a quick convergence and engineering accuracy is obtained in a much smaller calculation time as compared to the FEM, for both the strain and the potential formulations. Similar convergence behaviour is observed for a wide range of boundary conditions and different materials.

\section{Acknowledgements}

Elke Deckers is a Doctoral Fellow of the Fund for Scientific Research-Flanders (F.W.O.), Belgium. Furthermore, the authors gratefully acknowledge the European Commission for their support of the ITN Marie Curie project GA-214909 "MIDFREQUENCY - CAE Methodologies for Mid-Frequency Analysis in Vibration and Acoustics".

\section{Appendix A. List of symbols}

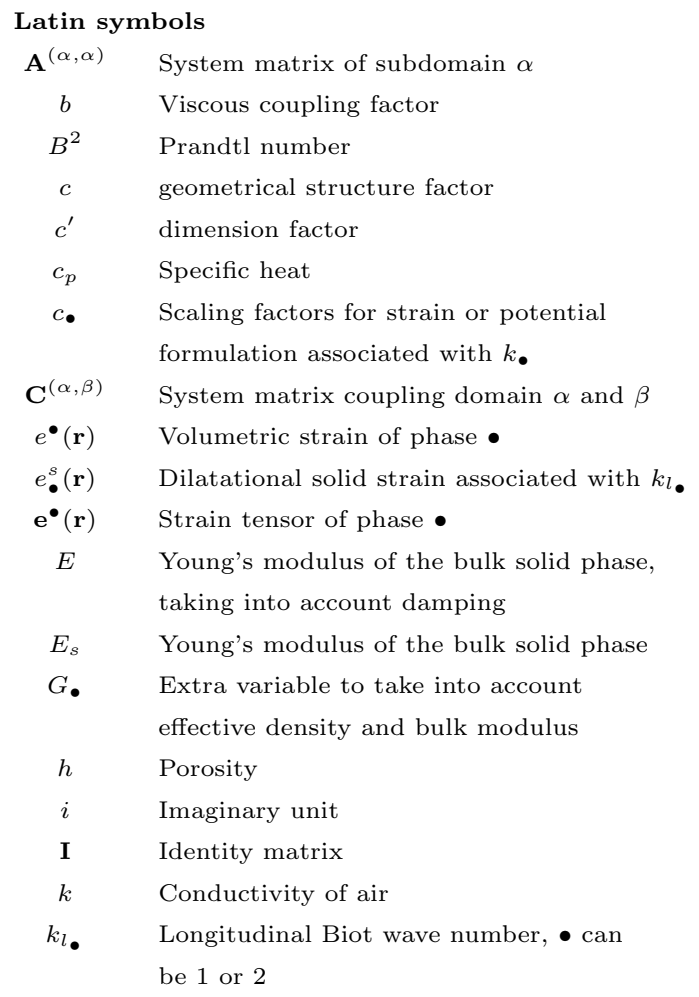

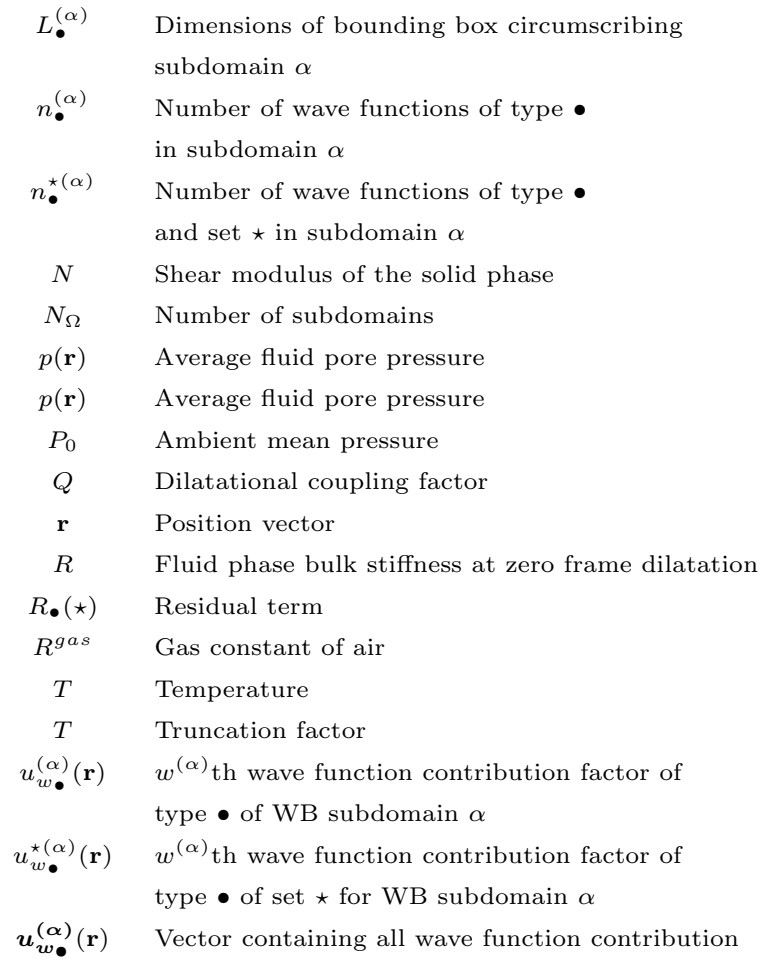




\begin{tabular}{|c|c|c|c|}
\hline$k_{t}$ & Rotational Biot wave number & & factors of type $\bullet$ \\
\hline$k_{* w \bullet}^{\star(\alpha)}$ & $\begin{array}{l}w \text { th } * \text {-component of the wave number associated } \\
\text { with set } \star \text { and type }\end{array}$ & $u_{\star}^{\bullet}(\mathbf{r})$ & $\begin{array}{l}\star \text {-component of the mean macroscopic } \\
\text { displacement of phase } \bullet\end{array}$ \\
\hline$K_{a}$ & Adiabatic Bulk modulus of the fluid & $\mathbf{u}^{\bullet}(\mathbf{r})$ & Mean macroscopic displacement vector of phase $\bullet$ \\
\hline$K_{f}$ & Effective Bulk modulus of the fluid & $w_{\bullet, \star}^{(\alpha)}$ & $\begin{array}{l}\text { Upper limit of number of wave functions in } \\
\text { truncation rule }\end{array}$ \\
\hline \multicolumn{4}{|c|}{ Greek symbols } \\
\hline$\alpha_{\infty}$ & Tortuosity & $\rho_{12}$ & Inertial mass coupling factor with inertial \\
\hline$\eta$ & Fluid dynamic viscosity & & dissipation \\
\hline$\eta_{l}$ & Loss factor & $\rho_{22}$ & Effective fluid mass density with inertial \\
\hline$\gamma$ & Ratio of specific heats & & dissipation \\
\hline$\gamma_{n}$ & Normal direction to a boundary & $\rho_{11}^{*}$ & Effective frame mass density with inertial \\
\hline$\gamma_{s}$ & Tangential direction to a boundary & & and viscous dissipation \\
\hline$\Gamma_{\bullet}$ & Boundary with conditions related to $\bullet$ & $\rho_{12}^{*}$ & Inertial mass coupling factor with inertial \\
\hline$\lambda$ & First Lamé constant of the solid phase & & and viscous dissipation \\
\hline$\tilde{\lambda}$ & Lamé parameter at zero acoustic pore pressure & $\rho_{22}^{*}$ & Effective fluid mass density with inertial \\
\hline$\Lambda$ & Viscous characteristic length & & and viscous dissipation \\
\hline$\Lambda^{\prime}$ & Thermal characteristic length & $\rho_{a}$ & Inertial coupling term \\
\hline$\mu_{\bullet}$ & Scaling constant between solid and fluid phase & $\sigma_{\star}^{\bullet}(\mathbf{r})$ & $\star$-component of the stress tensor of phase $\bullet$ \\
\hline$\nu$ & Poisson coefficient of the solid material & $\sigma^{\bullet}(\mathbf{r})$ & Stress tensor of phase $\bullet$ \\
\hline \multirow[t]{2}{*}{$\Phi_{w_{\bullet}}^{\star(\alpha)}(\mathbf{r})$} & $w_{\bullet}^{(\alpha)}$ th wave function of type $\bullet$ for WB & $\sigma$ & Static macroscopic flow resistivity \\
\hline & subdomain $\alpha$ of set $\star$ & $\varsigma_{\bullet}^{s}(\mathbf{r})$ & Symbol to indicate $e_{\bullet}^{s}(\mathbf{r})$ or $\varphi_{\bullet}^{s}(\mathbf{r})$ \\
\hline \multirow[t]{2}{*}{$\Phi_{w_{\bullet}}^{(\alpha)}(\mathbf{r})$} & $w_{\bullet}^{(\alpha)}$ th wave function of type $\bullet$ for WB & $\varphi_{\bullet}(\mathbf{r})$ & Scalar potential associated with $k_{l}$. \\
\hline & subdomain $\alpha$ & $\chi^{s}(\mathbf{r})$ & Symbol to indicate $\Omega^{s}(\mathbf{r})$ or $\psi^{s}(\mathbf{r})$ \\
\hline \multirow[t]{2}{*}{$\Phi_{w_{\bullet}}^{(\alpha)}(\mathbf{r})$} & Vector containing all wave function of type $\bullet$ & $\psi^{s}(\mathbf{r})$ & Vector potential of the solid phase \\
\hline & for WB subdomain $\alpha$ & $\omega$ & Circular frequency \\
\hline$\rho_{0}$ & Density of the interstitial fluid & $\Omega$ & Problem domain \\
\hline$\rho_{1}$ & Bulk frame mass density & $\Omega^{(\alpha)}$ & WB subdomain $\alpha$ \\
\hline$\rho_{2}$ & Bulk fluid mass density & $\Omega^{s}(\mathbf{r})$ & Rotational solid strain \\
\hline \multirow[t]{2}{*}{$\rho_{11}$} & Effective frame mass density & & \\
\hline & with inertial dissipation & & \\
\hline \multicolumn{4}{|c|}{ Other symbols } \\
\hline \multirow[t]{2}{*}{$\bullet^{(\alpha)}$} & Quantity or entity related to subdomain & $\nabla \times$ & Curl operator \\
\hline & WB subdomain $\alpha$ & $\nabla^{2}$ & Laplacian opererator \\
\hline$\hat{\bullet}$ & WB approximation of field variable $\bullet$ & $\frac{\partial \bullet}{\partial *}$ & Partial derivative \\
\hline$\tilde{\bullet}$ & WBM weighted residual test function & $\mathcal{L} \bullet$ & Linear differential operator \\
\hline$\nabla$ & Gradient operator & & \\
\hline
\end{tabular}

\section{Appendix B. Derivation of Biot parameters $Q, R, G(\omega)$ and $b$}

For acoustic applications $Q$ and $R$ can be approximated, respectively as $(1-h) K_{f}$ and $h K_{f}$. $K_{f}$ is the effective bulk modulus of the fluid in the pores. Two different formulations are used in this paper to obtain $K_{f}$ and $b$ : 
1. If it is assumed that the pores are identical parallel holes normal to the surface, then $G(\omega)=$ $G_{c}(s)[6]:$

$$
G_{c}(s)=-\frac{s}{4} \sqrt{-\mathrm{i}} \frac{J_{1}(s \sqrt{-\mathrm{i}})}{J_{0}(s \sqrt{-\mathrm{i}})}\left[1-\frac{2}{s \sqrt{-\mathrm{i}}} \frac{J_{1}(s \sqrt{-\mathrm{i}})}{J_{0}(s \sqrt{-\mathrm{i}})}\right]^{-1},
$$

where $J_{0}$ and $J_{1}$ are zero and first order Bessel functions of the first kind and $s$ is equal to:

$$
s=c \sqrt{\frac{8 \omega \rho_{0} \alpha_{\infty}}{\sigma h}},
$$

$c$ is the geometrical structure factor, taking into account the cross-sectional shape of the pores [6]. Then, $K_{f}$ is calculated as

$$
K_{f}=\frac{\gamma P_{0}}{\gamma-(\gamma-1)\left[1+\frac{8}{\mathrm{is} B^{2}} G_{c}\left(B^{2} s^{\prime}\right)\right]^{-1}},
$$

with $B^{2}$ the Prandtl number, $P_{0}$ the ambient mean pressure, $\gamma$ the ration of specific heats and $s^{\prime}$ defined as:

$$
s^{\prime}=\frac{1}{c^{\prime}} \sqrt{\frac{8 \omega \rho_{0} \alpha_{\infty}}{h \sigma}}
$$

where $c^{\prime}$ is a dimension factor.

2. Following the Johnson-Champoux-Allard approach [6], as for example used by Hörlin et al. $[18], G=G_{J}:$

$$
G_{J}(\omega)=\sqrt{1+\frac{4 \mathrm{i} \alpha_{\infty}^{2} \eta \rho_{0} \omega}{\sigma^{2} \Lambda^{2} h^{2}}}
$$

$\eta$ is the fluid dynamic viscosity and $\Lambda=\frac{1}{c} \sqrt{\frac{8 \alpha_{\infty} \eta}{h \sigma}}$ is the viscous characteristic length. In this approach, $K_{f}$ can be calculated as [6]:

$$
K_{f}=\frac{\gamma P_{0}}{\gamma-(\gamma-1)\left[1+\frac{8 \eta}{\mathrm{i} \Lambda^{\prime 2} B^{2} \omega \rho_{0}}\left(1+\mathrm{i} \rho_{0} \frac{\omega B^{2} \Lambda^{\prime 2}}{16 \eta}\right)^{1 / 2}\right]^{-1}},
$$

with $\Lambda^{\prime}=\frac{1}{c^{\prime}} \sqrt{\frac{8 \alpha_{\infty} \eta}{h \sigma}}$ the thermal characteristic length.

\section{Appendix C. WB system matrix construction}

This appendix gives the weighted residual formulation and explains how the WB system matrices are obtained. First all field variables are expanded in terms of wave functions. The transformations 
to the strain and potential formulation (11)-(12) are very similar. They only differ in the relative weighting between the longitudinal $\left(e_{\bullet}^{s}, \varphi_{\bullet}^{s}\right)$ and shear $\left(\Omega^{s}, \Psi^{s}\right)$ components of the decomposition. The transformations can be summarized as:

$$
\left\{\begin{array}{c}
u_{x}^{s(\alpha)}(\mathbf{r}) \\
u_{y}^{s(\alpha)}(\mathbf{r})
\end{array}\right\}=c_{l_{1}} \nabla \varsigma_{1}^{s(\alpha)}(\mathbf{r})+c_{l_{2}} \nabla \varsigma_{2}^{s(\alpha)}(\mathbf{r})+c_{t} \nabla \times \chi^{s(\alpha)}(\mathbf{r})
$$

where $c_{l_{1}}, c_{l_{2}}$ and $c_{t}$ depend on whether the displacements are transformed into strains (11) or potentials (12).

$$
\begin{array}{crrr}
\text { strain formulation : } & c_{l_{1}}=-\frac{1}{k_{l_{1}}^{2}} & c_{l_{2}}=-\frac{1}{k_{l_{2}}^{2}} & c_{t}=\frac{1}{k_{t}^{2}} \\
\text { potential formulation : } & c_{l_{1}}=1 & c_{l_{2}}=1 & c_{t}=1
\end{array}
$$

From here on, the formulations will be given in terms of the strains. The formulation in terms of potentials is obtained by replacing $e_{1}^{s}$ and $e_{2}^{s}$ by $\varphi_{1}^{s}$ and $\varphi_{2}^{s}$ and $\Omega^{s}$ by $\psi^{s}$ respectively and using the constants in $(\mathrm{C} .2 \mathrm{~b})$ corresponding to the transformation to potentials.

Each field variable $a^{(\alpha)}(\mathbf{r})$ can be written in terms of the strain fields by applying a corresponding differential operator:

$$
a^{(\alpha)}(\mathbf{r})=\mathcal{L}_{a}^{(\alpha)}\left[\begin{array}{c}
e_{1}^{s(\alpha)}(\mathbf{r}) \\
e_{2}^{s(\alpha)}(\mathbf{r}) \\
\Omega^{s(\alpha)}(\mathbf{r})
\end{array}\right]
$$

The differential operators for the normal and tangential displacements and the stresses in both the solid and the fluid phase are defined as follows:

$$
\begin{aligned}
\mathcal{L}_{u_{n}^{s}}^{(\alpha)} & =\left[\begin{array}{lll}
c_{l_{1}} \frac{\partial}{\partial \gamma_{n}^{(\alpha)}} & c_{l_{2}} \frac{\partial}{\partial \gamma_{n}^{(\alpha)}} & c_{t} \frac{\partial}{\partial \gamma_{s}^{(\alpha)}}
\end{array}\right] \\
\mathcal{L}_{u_{s}^{s}}^{(\alpha)} & =\left[\begin{array}{lll}
c_{l_{1}} \frac{\partial}{\partial \gamma_{s}^{(\alpha)}} & c_{l_{2}} \frac{\partial}{\partial \gamma_{s}^{(\alpha)}} & -c_{t} \frac{\partial}{\partial \gamma_{n}^{(\alpha)}}
\end{array}\right] \\
\mathcal{L}_{u_{n}^{f}}^{(\alpha)} & =\left[\begin{array}{lll}
c_{l_{1}} \mu_{l_{1}} \frac{\partial}{\partial \gamma_{n}^{(\alpha)}} & c_{l_{2}} \mu_{l_{2}} \frac{\partial}{\partial \gamma_{n}^{(\alpha)}} & c_{t} \mu_{t} \frac{\partial}{\partial \gamma_{s}^{(\alpha)}}
\end{array}\right] \\
\mathcal{L}_{\sigma_{n}^{s}}^{(\alpha)} & =\left[\begin{array}{lll}
c_{l_{1}}\left(2 N \frac{\partial^{2}}{\partial \gamma_{n}^{2(\alpha)}}+\left(\lambda+\mu_{l_{1}} Q\right) \nabla^{2}\right) & c_{l_{2}}\left(2 N \frac{\partial^{2}}{\partial \gamma_{n}^{2(\alpha)}}+\left(\lambda+\mu_{l_{2}} Q\right) \nabla^{2}\right) & c_{t} 2 N \frac{\partial^{2}}{\partial \gamma_{n}^{(\alpha)} \partial \gamma_{s}^{(\alpha)}}
\end{array}\right] \\
\mathcal{L}_{\sigma_{s}^{s}}^{(\alpha)} & =\left[\begin{array}{lll}
2 c_{l_{1}} \frac{\partial^{2}}{\partial \gamma_{n}^{(\alpha)} \partial \gamma_{s}^{(\alpha)}} & 2 c_{l_{2}} N \frac{\partial^{2}}{\partial \gamma_{n}^{(\alpha)} \gamma_{s}^{(\alpha)}} & c_{t} N\left(\frac{\partial^{2}}{\partial \gamma_{s}^{2 \alpha}}-\frac{\partial^{2}}{\partial \gamma_{n}^{2(\alpha)}}\right)
\end{array}\right] \\
\mathcal{L}_{\sigma^{f}}^{(\alpha)} & =\left[\begin{array}{lll}
c_{l_{1}}\left(Q+\mu_{l_{1}} R\right) \nabla^{2} & c_{l_{2}}\left(Q+\mu_{l_{2}} R\right) \nabla^{2} & 0
\end{array}\right]
\end{aligned}
$$


$\mu_{l_{1}}, \mu_{l_{2}}$ and $\mu_{t}$ are the ratios of the fluid and the solid displacements of the different wave types $[6]$ and are given by:

$$
\begin{aligned}
\mu_{l_{1}} & =\frac{(2 N+\lambda) k_{l_{1}}^{2}-\omega^{2} \rho_{11}^{*}}{\omega^{2} \rho_{12}^{*}-Q k_{l_{1}}^{2}} \\
\mu_{l_{2}} & =\frac{(2 N+\lambda) k_{l_{2}}^{2}-\omega^{2} \rho_{11}^{*}}{\omega^{2} \rho_{12}^{*}-Q k_{l_{2}}^{2}} \\
\mu_{t} & =-\frac{\rho_{12}^{*}}{\rho_{22}^{*}}
\end{aligned}
$$

The boundary and interface residuals of the porous material can be determined using the same differential operators. The mutual coupling between the three wave field components is entirely contained within the conditions specified along the boundaries and interfaces. For each subdomain $\Omega^{(\alpha)}$, the error residual functions are weighted with respect to some arbitrary weighting functions, indicated by $\tilde{\bullet}$ :

$$
\begin{array}{r}
\int_{\Gamma_{k i}^{(\alpha)} \cup \Gamma_{m i}^{(\alpha)}} \tilde{\sigma}_{n}^{s(\alpha)}(\mathbf{r}) R_{u_{n}^{s}}^{(\alpha)}(\mathbf{r}) d \Gamma+\int_{\Gamma_{k i}^{(\alpha)}} \tilde{\sigma}_{s}^{s(\alpha)}(\mathbf{r}) R_{u_{s}^{s}}^{(\alpha)}(\mathbf{r}) d \Gamma+\int_{\Gamma_{k i}^{(\alpha)} \cup \Gamma_{m i}^{(\alpha)}} \tilde{\sigma}^{f(\alpha)}(\mathbf{r}) R_{u_{n}^{f}}^{(\alpha)}(\mathbf{r}) d \Gamma \\
-\int_{\Gamma_{m e}^{(\alpha)}} \tilde{u}_{n}^{s(\alpha)}(\mathbf{r}) R_{\sigma_{n}^{s}}^{(\alpha)}(\mathbf{r}) d \Gamma-\int_{\Gamma_{m e}^{(\alpha)} \cup \Gamma_{m i}^{(\alpha)}} \tilde{u}_{s}^{s(\alpha)}(\mathbf{r}) R_{\sigma_{s}^{s}}^{(\alpha)}(\mathbf{r}) d \Gamma-\int_{\Gamma_{m e}^{(\alpha)}} \tilde{u}_{n}^{f(\alpha)}(\mathbf{r}) R_{\sigma^{f}}^{(\alpha)}(\mathbf{r}) d \Gamma \\
\quad+\sum_{\beta=1, \beta \neq \alpha}^{N_{\Omega}}\left(\int_{\Gamma_{I}^{(\alpha, \beta)}}\left(\tilde{\sigma}_{n}^{s^{(\alpha)}}(\mathbf{r}) R_{u_{n}^{s}}^{(\alpha, \beta)}(\mathbf{r})+\tilde{\sigma}_{s}^{s(\alpha)}(\mathbf{r}) R_{u_{s}^{s}}^{(\alpha, \beta)}(\mathbf{r})+\tilde{\sigma}^{f(\alpha)}(\mathbf{r}) R_{u_{n}^{f}}^{(\alpha, \beta)}(\mathbf{r})\right) d \Gamma\right) \\
-\sum_{\beta=1, \beta \neq \alpha}^{N_{\Omega}}\left(\int_{\Gamma_{I}^{(\alpha, \beta)}}\left(\tilde{u}_{n}^{s(\alpha)}(\mathbf{r}) R_{\sigma_{n}^{s}}^{(\alpha, \beta)}(\mathbf{r})+\tilde{u}_{s}^{s(\alpha)}(\mathbf{r}) R_{\sigma_{s}^{s}}^{(\alpha, \beta)}(\mathbf{r})+\tilde{u}_{n}^{f(\alpha)}(\mathbf{r}) R_{\sigma_{f}^{f}}^{(\alpha, \beta)}(\mathbf{r})\right) d \Gamma\right)=0
\end{array}
$$

This weighted residual formulation considers all kinds of possible boundary conditions. On each boundary of subdomain $\Omega^{(\alpha)}$ three residuals are imposed. The first six integrals result from the kinematic, mixed and mechanical boundary conditions. As can be seen, on every boundary $\Gamma_{\bullet}$, exactly the three residuals belonging to this boundary are evaluated. The two last terms in this equation result from interfaces between the considered subdomain $\Omega^{(\alpha)}$ and adjoining subdomains $\Omega^{(\beta)}$. It is supposed that the problem geometry can consist of $N_{\Omega}$ subdomains. Each two subdomains may or may not have an intermediate interface, which is taken into account by the summation. For every interface, in total 6 residuals are evaluated. One set is evaluated on the considered subdomain $\Omega^{(\alpha)}$, the other set is evaluated on the adjacent subdomain $\Omega^{(\beta)}$; so for every interface either the first set or the second set is taken into account. The weighting functions $\tilde{a}$ (with $a=u_{n}^{s}, u_{s}^{s}, u_{n}^{f}, \sigma_{n}^{s}, \sigma_{s}^{s}, \sigma^{f}$ ) 
are expressed in terms of the same set of wave functions as used in the field variable expansions:

$$
\tilde{a}=\mathcal{L}_{a}\left[\begin{array}{c}
\tilde{e}_{1}^{s}(\mathbf{r}) \\
\tilde{e}_{2}^{s}(\mathbf{r}) \\
\tilde{\Omega}^{s}(\mathbf{r})
\end{array}\right]
$$

with

$$
\begin{gathered}
\tilde{e}_{1}^{s}(\mathbf{r})=\Phi_{\mathbf{w}_{\mathbf{e}_{1}^{s}}}^{(\alpha)}(\mathbf{r}) \tilde{\mathbf{u}}_{\mathbf{w}_{\mathbf{e}_{1}^{s}}}^{(\alpha)} \\
\tilde{e}_{2}^{s}(\mathbf{r})=\boldsymbol{\Phi}_{\mathbf{w}_{\mathbf{e}_{2}^{s}}^{s}}^{(\alpha)}(\mathbf{r}) \tilde{\mathbf{u}}_{\mathbf{w}_{\mathbf{e}_{2}^{s}}}^{(\alpha)} \\
\tilde{\Omega}^{s}(\mathbf{r})=\Phi_{\mathbf{w}_{\Omega^{\mathbf{s}}}}^{(\alpha)}(\mathbf{r}) \tilde{\mathbf{u}}_{\mathbf{w}_{\Omega^{s}}}^{(\alpha)}
\end{gathered}
$$

Substituting the field variable expansions and the weighting function expansions into the weighted residual formulation for subdomain $\Omega^{(\alpha)}(\mathbf{r})$, yields an algebraic equation which links together the wave function contribution factors of subdomain $\Omega^{(\alpha)}$ and those of the adjacent subdomains. This procedure is repeated for each subdomain. By enforcing that this equation should hold for any weighting function and thus for every possible combination of $\tilde{u}_{w_{\bullet_{i}}}^{(\alpha)}$ results in a fully populated, complex and generally nonsymmetric system of equations:

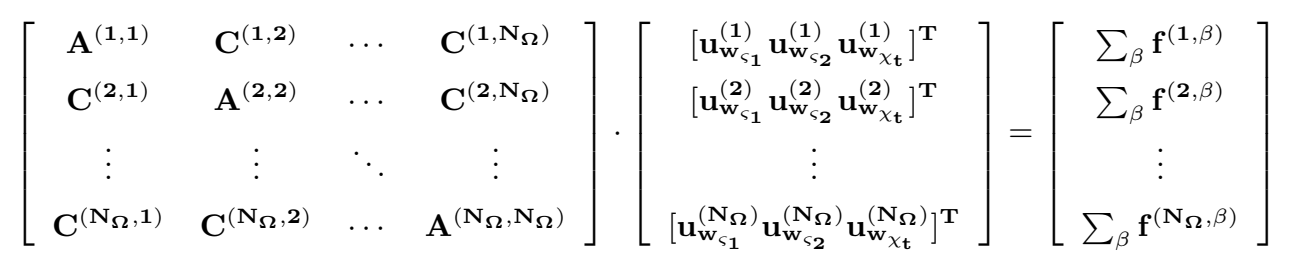

The matrices $\mathbf{A}^{(\bullet \bullet \bullet)}$ are the system matrices, the matrices $\mathbf{C}^{(\bullet \bullet \bullet)}$ are the coupling matrices, linking the wave functions of two adjacent subdomains and $\mathbf{f}^{(\bullet)}$ are the loading vectors resulting from nonhomogeneous kinematic, mechanical or mixed boundary conditions. General expressions to evaluate these WB matrices are given in [38]. The solution of these equations yields the contribution factor of each wave function in the solution expansion.

[1] T. Courtois, C. Bertolini, J. Ochs, A Procedure for Efficient Trimmed Body FE Simulations, Based on a Transfer Admittance Model of the Sound Package, In Proceedings of the 6th Styrian Noise, Vibration $\&$ Harshness Congress, Graz, Austria, 2010.

[2] W.R. Graham, Boundary layer induced noise in aircraft, part II: the trimmed flat plate model, Journal of Sound and Vibration 192 (1996) 121-138. 
[3] M. A. Biot, The theory of propagation of elastic waves in a fluid-saturated porous solid. I. Low frequency range. II. Higher frequency range, J. Acoust. Soc. Am. 28 (1956) 168-191.

[4] D. L. Johnson, J. Koplik, R. Dashen, Theory of dynamic permeability and tortuosity in fluidsaturated porous media, J. Fluid Mech. 176 (1987) 379-402.

[5] Y. Champoux, J.F. Allard, Dynamic tortuosity and bulk modulus in air-saturated porous media, J. Appl. Phys. 70 (1991) 1975-1979.

[6] J.F. Allard, N. Atalla, Propagation of Sound in Porous Media: Modeling Sound Absorbing Materials, John Wiley \& Sons, West Sussex, United Kingdom 2009.

[7] T. Yamamoto, S. Maruyama, K. Terada, K. Izui and S. Nishiwaki, A generalized macroscopic model for sound-absorbing poroelastic media using the homogenization method, Comput. Methods Appl. Mech. Engrg. 200 (2011) 251-264.

[8] J. E. Santos, J. G. Rubino and C. L. Ravazzoli, A numerical upscaling procedure to estimate effective plane wave and shear moduli in heterogeneous fluid-saturated poroelastic media, Comput. Methods Appl. Mech. Engrg. 198 (2009) 2067-2077.

[9] M.-E. Delany, E.N. Bazley, Acoustical properties of fibrous materials, Appl. Acoust. 3 (1970) 105-116.

[10] J.F. Allard, C. Depollier, P. Rebillard, W. Lauriks, A. Cops, Inhomogeneous Biot waves in layered media, J. Appl. Phys. 66 (1989) 2278-2284.

[11] K. Attenborough, Acoustical characteristics of rigid fibrous absorbents and granular media, J. Acoust. Soc. Am. 73 (1998) 785-799.

[12] Y.J. Kang, J.S. Bolton, Finite element modeling of isotropic elastic porous materials coupled with acoustical finite elements, J. Acoust. Soc. Am. 98 (1995) 635-643.

[13] R. Panneton, N. Atalla, An efficient finite element scheme for solving the three dimensional poro-elasticity problems in acoustics, J. Acoust. Soc. Am. 101 (1997) 3287-3297.

[14] N. Atalla, R. Panneton, P. Debergue, Enhanced weak integral formulation for the mixed ( $\underline{u}, \underline{p})$ poroelastic equations, J. Acoust. Soc. Am. 109 (2001) 3065-3068. 
[15] P. Debergue, R. Panneton, N. Atalla, Boundary conditions for the weak formulation of the mixed (u,p) poroelasticity problem, J. Acoust. Soc. Am. 106 (1999) 2383-2390.

[16] N. Dauchez, S. Sahraoui, N. Atalla, Convergence of poroelastic finite elements based on Biot displacement formulation, J. Acoust. Soc. Am. 109 (2001) 33-40.

[17] O. Dazel, B. Brouard, C. Depollier, S. Griffiths, An alternative Biot's displacement formulation for porous materials, J. Acoust. Soc. Am. 121 (2007) 3509-3516.

[18] N.-E. Hörlin, M. Nordström, P. Göransson, A 3-D hierarchical FE formulation of Biot's equations for elasto-acoustic modelling of porous media, J. Sound Vib. 245 (2001) 633-652.

[19] S. Rigobert, N. Atalla. F.C. Sgard, Investigation of the convergence of the mixed displacementpressure poroelastic materials using hierarchical elements, J. Acoust. Soc. Am. 114 (2003) 26072617.

[20] O. Dazel, F. Sgard, C.H. Lamarque and N. Atalla, An extension of complex modes for the resolution of finite-element poroelastic problems, J. Sound Vib. 253 (2002) 421-445.

[21] O. Dazel, F. Sgard and C.H. Lamarque, Application of generalized complex modes to the calculation of the forced response of three-dimensional poroelastic materials, J. Sound Vib. 268 (2003) 555-580.

[22] A. Duval., J. Baratier, C. Morgenstern, L. Dejaeger, N. Kobayashi and H. Yamaoka, Trim FEM simulation of a dash and floor insulator cut out modules with structureborne and airborne excitations, in Proceedings of euronoise 2008, Paris, France, 3779-3784.

[23] O. Tanneau, P. Lamary, Y. Chevalier, A boundary element method for porous media, J. Acoust. Soc. Am. 120 (2006) 1239-1251.

[24] S. Ghinet and N. Atalla, Vibro-acoustic behaviour of flat sandwich behaviour of multi-layer orthotropic panels, Transactions of Canadian Acoustics 30 (2002) 72-73.

[25] N. Atalla, F. Sgard, C.K. Amedin, On the modeling of sound radiation from poroelastic materials, J. Acoust. Soc. Am. 120 (2006) 1990-1995.

[26] D. Rhazi and N. Atalla, Transfer matrix modeling of the vibroacoustic response of multimaterials structures under mechanical excitation, J. Sound Vib. 329 (2010) 2532-2546. 
[27] W. Desmet, A wave based prediction technique for coupled vibro-acoustic analysis, Katholiek Universiteit Leuven, PhD thesis 98D12, Leuven, 1998.

[28] E. Trefftz, Ein Gegenstück zum Ritzschen Verfahren, In Proceedings of the 2nd International Congress on Applied Mechanics, Zurich, Switzerland, pp.131-137, 1926.

[29] B. Pluymers, B. Van Hal, D. Vandepitte, W. Desmet, Trefftz-based methods for time-harmonic acoustics, Arch. Comput. Methods Eng., 14 (2007) 343-381.

[30] B. Van Hal, W. Desmet, D. Vandepitte, A coupled finite element-wave based approach for the steady state dynamic analysis of acoustic systems, J. Comput. Acoust., 11 (2003) 285-303.

[31] B. Bergen, B. Van Genechten, D. Vandepitte and W. Desmet, An efficient Trefftz-based method for three-dimensional Helmholtz problems in unbounded domains, Comput. Modeling in Eng. Sciences 61 (2010) 155-175.

[32] R. Lanoye, G. Vermeir, W. Lauriks, F. Sgard, W. Desmet, Prediction of the sound field above a patchwork of absorbing materials, J. Acoust. Soc. Am. 123 (2008) 793-802.

[33] C. Vanmaele, D. Vandepitte, W. Desmet, An efficient wave based prediction technique for plate bending vibrations, Comput. Methods Appl. Mech. Engrg. 196 (2007) 3178-3189.

[34] C. Vanmaele, D. Vandepitte, W. Desmet, An efficient wave based prediction technique for dynamic plate bending problems with corner stress singularities, Comput. Methods Appl. Mech. Engrg. 198 (2009) 2227-2245.

[35] B. Pluymers, W. Desmet, D. Vandepitte, P. Sas, On the Use of a Wave Based Prediction Technique for Steady-State Structural-Acoustic Radiation Analysis, J. Comput. Model. Eng. \& Sci. 7 (2005) 173-184.

[36] B. Pluymers, W. Desmet, D. Vandepitte, P. Sas, Application of an efficient wave based prediction technique for the analysis of vibro-acoustic radiation problems, J. Comput. Appl. Math. 168 (2004) 353-364.

[37] B. Van Genechten, D. Vandepitte, W. Desmet, A Trefftz-based numerical modelling framework for Helmholtz problems with complex multiple scatterer configurations, J. Comput. Phys. 229 (2010) 6623-6643. 
[38] B. Van Genechten, K. Vergote, D. Vandepitte, W. Desmet, A Multi-Level Wave Based numerical modelling framework for the steady-state dynamic analysis of bounded Helmholtz problems with multiple inclusions, Comput. Methods Appl. Mech. Engrg. 199 (2010) 1881-1905.

[39] B. Van Genechten, D. Vandepitte, W. Desmet, A direct hybrid Finite Element - Wave based modelling technique for efficient coupled vibro-acoustic analysis, Comput. Methods Appl. Mech. Engrg. 200 (2011) 742-764.

[40] R. Burridge, J.B. Keller, Poroelasticity equations derived from microstructure, J. Acoust. Soc. Am. 70 (1981) 1140-1146.

[41] S.R. Pride, A.F. Gangi, F.D. Morgan, Deriving the equations of motion for porous isotropic media, J. Acoust. Soc. Am. 92 (1992) 3278-3290.

[42] M.A. Biot, D.G. Willis, The elastic coefficients of consolidation, J. Appl. Mechanics 34 (1957) 594-601.

[43] J.S. Bolton, N.-M. Shiau, Y.J. Kang, Sound transmission through multi-panel structures lined with elastic porous materials, J. Sound Vib. 191 (1996) 317-347.

[44] H.L.F. Helmholtz, Über Integrale der hydrodynamischen Gleichungen, welch den Wirbelbewegungen entsprechen, Crelles Journal 55 (1958) pp. 25-55.

[45] T. Huttunen, P. Gamallo, R.J. Astley, Comparison of two wave element methods for the Helmholtz problem, Commun. Numer. Methods Eng. 25 (2009) 35-52.

[46] A.P. Zielinski and I. Herrera, Trefftz method: fitting boundary conditions, Int. J. Numer. Methods Eng. 24 (1987) 871-891.

[47] B. Van Hal, Automation and performance optimization of the wave based method for interior structural-acoustic problems, K.U.Leuven, division PMA, PhD. thesis 2004D07, 2004.

[48] E. Deckers, B. Drofmans, B. Van Genechten, B. Bergen, D. Vandepitte, W. Desmet, Splinebased boundaries: a first step towards generic geometic domain descriptions for efficient midfrequency acoustic analysis using the Wave Based Method, J. Comput. Appl. Math. 235 (2011) 2679-2693. 
[49] B. Pluymers, Wave Based modelling methods for steady-state vibro-acoustics, K.U.Leuven, division PMA, PhD. thesis 2006D04, Leuven, 2006.

[50] D. Huybrechs, Multiscale and hybrid methods for the solution of oscillatory integral equations, PhD. thesis, Katholieke Universitiet Leuven, Departement Computerwetenschappen, Leuven, Belgium. 